\title{
The lagRST Model: a Turbulence Model for Non-Equilibrium Flows
}

\author{
Randolph P. Lillard* \\ A. Brandon Oliver ${ }^{\dagger}$ \\ NASA Johnson Space Center \\ Houston, TX 77058 \\ Michael E. Olsen ${ }^{\ddagger}$ \\ NASA Ames Research Center \\ Moffet Field, CA 94035
Gregory A. Blaisdell§ and Anastasios S. Lyrintzis
School of Aeronautics and Astronautics
Purdue University
West Lafayette, IN 47907

\begin{abstract}
This study presents a new class of turbulence model designed for wall bounded, high Reynolds number flows with separation. The model addresses deficiencies seen in the modeling of nonequilibrium turbulent flows. These flows generally have variable adverse pressure gradients which cause the turbulent quantities to react at a finite rate to changes in the mean flow quantities. This "lag" in the response of the turbulent quantities can't be modeled by most standard turbulence models, which are designed to model equilibrium turbulent boundary layers. The model presented uses a standard 2-equation model as the baseline for turbulent equilibrium calculations, but adds transport equations to account directly for non-equilibrium effects in the Reynolds Stress Tensor (RST) that are seen in large pressure gradients involving shock waves and separation. Comparisons are made to several standard turbulence modeling validation cases, including an incompressible boundary layer (both neutral and adverse pressure gradients), an incompressible mixing layer and a transonic bump flow. In addition, a hypersonic Shock Wave Turbulent Boundary Layer Interaction with separation is assessed along with a transonic capsule flow. Results show a substantial improvement over the baseline models for transonic separated flows. The results are mixed for the SWTBLI flows assessed. Separation predictions are not as good as the baseline models, but the over prediction of the peak heat flux downstream of the reattachment shock that plagues many models is reduced.
\end{abstract}

\section{Greek Symbols}

$\alpha \quad$ Angle of attack or turbulence modeling constant

$\beta \quad$ Clauser's pressure gradient parameter, turbulence modeling constant

$\delta \quad$ Boundary layer thickness, $\mathrm{m}$

$\delta^{*} \quad$ Displacement thickness, $\mathrm{m}$

$\epsilon \quad$ Dissipation per unit mass, $\mathrm{m}^{2} / \mathrm{s}^{3}$

$\kappa \quad$ Turbulence modeling constant

$\mu \quad$ Dynamic viscosity, $\mathrm{kg} / \mathrm{m} \cdot \mathrm{s}$

$\nu \quad$ Kinematic viscosity, $\mathrm{m}^{2} / \mathrm{s}$

$\omega \quad$ Specific dissipation, $s^{-1}$

*Aerospace Engineer, NASA JSC.

$\dagger$ Aerospace Engineer, NASA JSC, Ph.D. Candidate, Purdue University.

${ }^{\ddagger}$ Research Scientist, NASA Ames Research Center, Associate Fellow AIAA.

$\S$ Associate Professor, Associate Fellow AIAA.

TProfessor, Associate Fellow AIAA. 


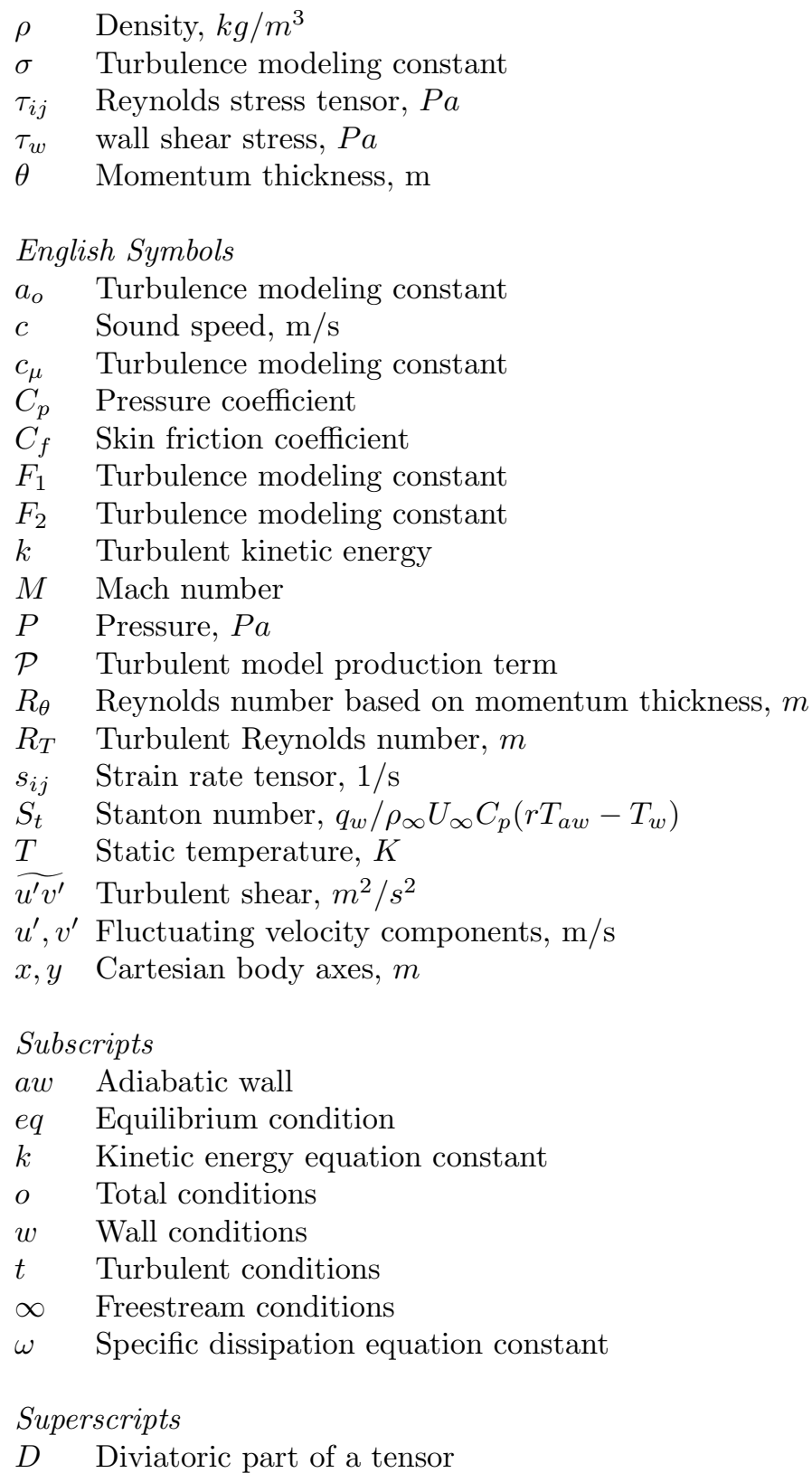

\section{Introduction}

Computational predictions of the aerodynamic and aerothermodynamic environments a space vehicle encounters are essential for the design and operation of the vehicle. As computer speeds continue to increase, Computational Fluid Dynamics (CFD) can be used to not only predict the flowfield around simple configurations, but also complex vehicles. The advances in computer speeds have given rise to advances in the modeling of turbulent flows using Direct Numerical Simulations (DNS) and Large Eddy Simulations (LES). For complex configurations, DNS or even LES are still impractical because of the grid spacing requirements and the need for time-accurate solutions. Although DNS and LES employ a more physics based representation of the fluid dynamics occurring, the Reynolds Averaged Navier Stokes (RANS) Equations solved along with a turbulence model are still a valuable tool for aerodynamic analysis.

One difficulty with current one and two-equation turbulence models is the inability to account directly for non-equilibrium effects such as those encountered in large pressure gradients involving separation and shockwaves. Current turbulence models such as Spalart's one-equation model, ${ }^{1}$ the classic $k-\epsilon^{2,3}$ and 
Wilcox's $k-\omega^{4}$ two-equation models have been designed and tuned to accurately predict equilibrium flows such as zero-pressure gradient boundary- layers and free shear layers. Application to more complex flows can be problematical at best. Although there have been many attempts to modify or correct basic one- and two-equation models, most of these attempts have been only marginally successful in predicting complex flows.

More complex models such as Reynolds stress models have been investigated extensively, primarily for relatively simple flows but also for complex flows. In most cases these models give somewhat better predictions than the simpler one and two equation models, but for complex flows they do not perform much better than the simpler models. Rizzetta ${ }^{5}$ evaluated three popular explicit algebraic Reynolds-stress (EARS) models for separated supersonic flows, a shock impinging on turbulent boundary layer and a compression ramp. He compared these results to the $\mathrm{k}-\epsilon$ model. The results consistently showed that the EARS models offered little improvement over standard RANS approaches using the Boussinesq approximation. Viti et al. ${ }^{6}$ showed similar results for the Reynolds stress-transport turbulence models.

One theoretical advantage of Reynolds stress models is that they directly account for non-equilibrium effects in the sense that the Reynolds stresses do not respond instantaneously to changes to the strain rate but more realistically lag them in time and/or space. Unfortunately, the Reynolds stress models are usually considerably more complicated and numerically stiff than the one- and two- equation models, and this has prevented their wide application for complex flows.

In this paper we introduce a new turbulence model designed to account for non-equilibrium effects without invoking the full formalism of the Reynolds stress models. The idea is an extension of Olsen and Coakley's lag model, ${ }^{7}$ which took a baseline two-equation model and coupled it with a third (lag) equation to model the non-equilibrium effects for the eddy viscosity. The third equation was designed to predict the equilibrium eddy viscosity in equilibrium flows. The proposed lagRST model uses the same lag equation idea, but directly lags the Reynolds stress tensor components instead of the eddy viscosity. The Boussinesq approximation sets the equilibrium values of each Reynolds stress tensor component that the lag equations will drive the value towards.

We show results obtained with two Reynolds stress lag models based on the Wilcox k- $\omega$ model $^{4}$ and Menter's SST model, ${ }^{8,9}$ respectively. Applications to four flows are given including an incompressible flat plate flow, an incompressible mixing layer, ${ }^{10}$ an essentially incompressible adverse pressure gradient flow with separation, ${ }^{11}$ and a transonic bump flow ${ }^{12}$ with a shock wave and separation. In addition, aerodynamic comparisons will be made to Apollo wind tunnel data. ${ }^{13}$ We also are looking for a compressible shock wave turbulent boundary layer interaction case to assess. Results using the new model are compared with results obtained with Spalart's model ${ }^{1}$ and Menter's k- $\omega$ SST model. Results obtained with the new model show encouraging improvements over results obtained with the other models.

\section{I.A. Non-Equilibrium Turbulence}

Several reviews have been done ${ }^{14-16}$ assessing the accuracy of one- and two-equation turbulence models in the prediction of SWTBLI. The reviews show that one- and two-equation models poorly predict the starting position of the adverse pressure gradient flow, over predict the surface pressure within separation, and over predict skin friction and heat transfer at the re-attachment point. It is proposed that a main cause of this inaccuracy of the predictions in the separated regions is the effect of flow history. In other words, the Boussinesq approximation relates mean strain rates directly to the Reynolds stress tensor (RST). Thus any change in the strain rate is instantly accounted for in the RST. In reality, there is a time lag between changes in the strain rate and changes in the RST when the turbulence is not in equilibrium. Turbulent flows deviate from equilibrium conditions when large adverse pressure gradients are present. The standard one- and two-equation turbulence models have been designed and tuned to accurately predict equilibrium flows, such as zero-pressure gradient boundary layers and free shear layers.

Clauser ${ }^{17}$ defined a boundary layer to be in equilibrium if the pressure gradient parameter

$$
\beta=\frac{\delta^{*}}{\tau_{w}} \frac{d P_{o}}{d x}
$$

is a constant. When boundary layers are in equilibrium, they are self-preserving, i.e. when properly scaled, the wake portions of the velocity profile for two different boundary layers with the same value of $\beta$ are identical, even if their Reynolds numbers are different. Equilibrium boundary layers have the characteristic 
that the turbulent time scales are much smaller than the mean flow time scales so that the turbulence can react quickly to these slowly changing mean flows.

\section{I.B. lagRST Model}

Modeling nonequilibrium turbulent effects have been attempted by many researchers. An overview of many of these models can be found in Lillard. ${ }^{18}$ One of these models, the Lag model of Olsen and Coakley ${ }^{7}$ and Olsen et al. ${ }^{19}$ has provided promising results for nonequilibrium flows. It is formed by taking a baseline two-equation model and coupling it with a third equation (lag equation) to model the nonequilibrium effects in the eddy viscosity. This class of models essentially introduces a lag into the response of the eddy viscosity to rapid changes in the mean flowfield, which is the character of the response seen experimentally. By lagging the third equation it provides the turbulence model an extra degree of freedom without affecting accurate equilibrium flow predictions.

The lagRST model was designed to build upon the success of the Lag model by using the same baseline formulation, but directly solving for the Reynolds stresses, relaxing each stress independently along a streamline. The equilibrium state is defined by transport equations for the turbulent kinetic energy, k, and the specific dissipation rate, $\omega$. These are shown in equations 3 and 4 , respectively. The lag equation for turbulent viscosity is then replaced with a similar relation for the Reynolds stress tensor, $\tau_{i j}$, defined as

$$
\tau_{i j}=-\bar{\rho} \widetilde{u_{i}^{\prime} v_{j}^{\prime}}
$$

Equation 5 details the lag equation for $\tau_{i j}$. A separate equation is added for each Reynolds stress (this becomes six equations since the tensor is symmetric).

$$
\begin{aligned}
\frac{\partial \rho k}{\partial t}+\frac{\partial}{\partial x_{k}}\left(\rho u_{k} k-\left[\mu+\sigma_{k} \mu_{t}\right] \frac{\partial k}{\partial x_{k}}\right) & =\mathcal{P}_{k}-\epsilon_{k} \\
\frac{\partial \rho \omega}{\partial t}+\frac{\partial}{\partial x_{k}}\left(\rho u_{k} \omega-\left[\mu+\sigma_{\omega} \mu_{t}\right] \frac{\partial \omega}{\partial x_{k}}\right) & =\mathcal{P}_{\omega}-\epsilon_{\omega} \\
\frac{\partial \tau_{i j}}{\partial t}+\frac{\partial}{\partial x_{k}}\left(\tau_{i j} \tilde{u}_{k}\right) & =a_{o} \omega\left(\tau_{i j_{e q}}-\tau_{i j}\right)
\end{aligned}
$$

where:

$$
\begin{array}{rlrl}
\nu_{t_{e q}} & =k / \omega & \mathcal{P}_{\omega} & =\alpha \rho S^{2} \\
\mathcal{P}_{k} & =\tau_{i j} s_{i j} & \epsilon_{\omega} & =\beta \rho \omega^{2} \\
\epsilon_{k} & =\beta^{*} \rho k w & S & =\sqrt{2\left(s_{i j} s_{i j}-s_{k k}^{2} / 3\right)} \\
\tau_{i j_{e q}} & =-\rho\left(\frac{2}{3} k \delta_{i j}-\nu_{t_{e q}}\left(2 s_{i j}-\frac{2}{3} s_{k k} \delta_{i j}\right)\right) & s_{i j} & =\frac{1}{2}\left(\frac{\partial u_{i}}{\partial x_{j}}+\frac{\partial u_{j}}{\partial x_{i}}\right)
\end{array}
$$

with parameters

$$
\begin{aligned}
a_{0} & =0.35 & \beta & =0.075 & \sigma_{k} & =1.5 \\
\alpha & =5 / 9 & \beta^{*} & =0.9 & \sigma_{e} & =0.5 .
\end{aligned}
$$

The equilibrium values of the Reynolds stresses are determined by using the Boussinesq approximation and solving for $\tau_{i j_{e q}}$.

$$
\tau_{i j_{e q}}=-\rho\left[\frac{2}{3} k \delta_{i j}-\nu_{t_{e q}}\left(2 s_{i j}-\frac{2}{3} s_{k k} \delta_{i j}\right)\right]
$$

\section{I.B.1. Lagging the Reynolds Stress Tensor using Menter's SST}

A version of the LagRST model using Menter's SST model ${ }^{9}$ to provide the equilibrium values of $k$ and $\omega$ was also implemented. Equations 7 and 8 provide the transport equations for the equilibrium quantities. 


$$
\begin{aligned}
\frac{D \rho k}{D t}= & \tau_{i j} \frac{\partial u_{i}}{\partial x_{j}}-\beta^{*} \rho \omega k+\frac{\partial}{\partial x_{j}}\left[\left(\mu+\sigma_{k} \mu_{t}\right) \frac{\partial k}{\partial x_{j}}\right] \\
\frac{D \rho \omega}{D t}= & \frac{\gamma}{\nu_{t}} \tau_{i j} \frac{\partial u_{i}}{\partial x_{j}}-\beta \rho \omega^{2}+\frac{\partial}{\partial x_{j}}\left[\left(\mu+\sigma_{\omega} \mu_{t}\right) \frac{\partial \omega}{\partial x_{j}}\right] \\
& +2 \rho\left(1-F_{1}\right) \sigma_{\omega 2} \frac{1}{\omega} \frac{\partial k}{\partial x_{j}} \frac{\partial \omega}{\partial x_{j}}
\end{aligned}
$$

The modeling constants and blending functions used were not modified and therefore can be found in Menter. ${ }^{9}$ The turbulent eddy viscosity was calculated the standard way, Where $\nu_{t}$ is defined as

$$
\nu_{t_{e q}}=\frac{a_{1} k}{\max \left(a_{1} \omega ; \Omega F_{2}\right)}
$$

This was then substituted into equation 6 to obtain $\tau_{i j_{e q}}$.

\section{I.C. Summary of Proposed Models}

Two Reynolds stress lag models were implemented into OVERFLOW v2.0aa and v2.2c. Table 2 details the name of the model and how the equilibrium value are obtained. The lagRST model uses the $k-\omega$ model to define equilibrium values while the lagRSTSST model uses the SST model. Both models use the same transport equation for $\tau_{i j}$, which was already shown in equation 5 . The lagRST model per time step was

Table 1. Lag variables

\begin{tabular}{|c|c|c|}
\hline Model (Designator) & lag variable & equilibrium variable \\
\hline LagRST (905) & $\tau_{i j}=-\bar{\rho} \widetilde{u_{i}^{\prime} v_{j}^{\prime}}$ & $\begin{array}{c}\tau_{i j_{e q}}=-\rho\left(\frac{2}{3} k_{e q} \delta_{i j}-\nu_{t_{e q}}\left(2 s_{i j}-\frac{2}{3} s_{k k} \delta_{i j}\right)\right) \\
\nu_{t_{e q}}=\frac{k_{e q}}{\omega_{e q}}, \text { defined from } k-\omega\end{array}$ \\
\hline lagRSTSST (909) & $\tau_{i j}=-\bar{\rho} \widetilde{u_{i}^{\prime} v_{j}^{\prime}}$ & $\begin{array}{c}\tau_{i j_{e q}}=-\rho\left(\frac{2}{3} k_{e q} \delta_{i j}-\nu_{t_{e q}}\left(2 s_{i j}-\frac{2}{3} s_{k k} \delta_{i j}\right)\right) \\
\nu_{t_{e q}}=\frac{a_{1} k_{e q}}{\max \left(a_{1} \omega_{e q} ; \Omega F_{2}\right)}, \text { defined from SST }\end{array}$ \\
\hline
\end{tabular}

$35 \%$ slower than the SA model and $20 \%$ slower than the SST.

\section{I.C.1. Modeling Constants}

During the course of this study, it was discovered that an exhaustive study of the effect of $\sigma_{k}$ had not been done in the original Lag model formulation. Since the formulation of the original Lag model, three values had been proposed for the value of $\sigma_{k}, 0.5,0.8$ and 1.5. Although previously thought to only be a cosmetic change, the value of $\sigma_{k}$ makes a substantial difference near the edge of the boundary layer. In addition, $a_{o}$ will be assessed for values of 0.35 and 0.2 . The value of 0.35 is the standard defined by Olsen and Coakley ${ }^{7}$ and Olsen et al. ${ }^{19}$

\section{I.D. Numerical Method}

The lagRST and lagRSTSST models were implemented into version 2.0aa and 2.2c of the OVERFLOW code. OVERFLOW ${ }^{20,21}$ is a structured (overset) grid, Navier-Stokes flow solver. It uses a finite-difference formulation, with flow quantities stored at the grid nodes. OVERFLOW has central- and Roe upwinddifference options, and uses a diagonalized, implicit approximate factorization scheme for time advancement. Local timestepping, multigrid techniques and grid sequencing are all used to accelerate convergence to a steady state. The standard turbulence models used by the OVERFLOW code are the one-equation SA model and the two-equation SST model. Other models available are the Baldwin-Lomax, k- $\epsilon$ and the k- $\omega$. SA and SST are used for general aerodynamic applications within the OVERFLOW community. The full Navier Stokes equations were solved for all solutions. 


\section{I.E. Results with Similar Models}

Hamlington and Dahm ${ }^{22,23}$ have derived a model similar to our proposed lagRST model in which they use the Reynolds anisotropy tensor (similar to the version discussed above). However, instead of numerically solving the lag equation, they solve the lag equation along a streamline, reducing it to an ordinary differential equation. They assessed several homogenous flows and have done work on flat plates and the interaction of an impinging oblique shock wave with a turbulent boundary layer.

Churchfield and Blaisdell ${ }^{24}$ used a similar definition of the lagRST model ${ }^{25}$ discussed herein, but used the $2006 k-\omega$ formulation to define $\nu_{t e q}$. Churchfield and Blaisdell ${ }^{26,27}$ then went on to use a previous version of the lagRST formulation incorporated in the OVERFLOW code provided by Lillard ${ }^{25}$ to assess wingtip vortex flow.

\section{Results}

Four test cases were chosen, to cover a broad range of flow physics while keeping the modeling complications to a minimal level. These cases were chose in order to execute the following:

1. Verify the implementation of the lagRST and lagRSTSST models.

2. Confirm the value of the lag constant, $a_{o}$, previously derived by Olsen and Coakley ${ }^{28}$ as 0.35 .

3. Assess the sensitivity of the models to $\sigma_{k}$ and confirm the value obtained by Olsen et al. ${ }^{29}$

4. Assess the performance of the lagRST and lagRSTSST models in predicting basic aerodynamic flows, such as turbulent boundary layer flow, mixing layers, and simple separated flows.

In addition, several other turbulence models were run for comparison purposes including the SpalartAllmaras, k- $\omega$ 1998, SST, and Lag models. Although the $k-\omega$ model is not a standard model anymore due to known freestream dependencies, it will be assessed here for comparison because it is the base model for the Lag model and the majority of the lagRST models. Table 2 lists the set of turbulence models that are referred to by number instead of name in some of the upcoming figures. The associated number is also the turbulence model designator within the OVERFLOW code.

Table 2. Table of turbulence models and their associated reference numbers in the OVERFLOW code

\begin{tabular}{|c|c|}
\hline Model Number & Turbulence Model \\
\hline 102 & $\mathrm{SA}$ \\
202 & $\mathrm{k}-\omega$ \\
203 & $\mathrm{SST}$ \\
304 & $\mathrm{Lag}$ \\
903 & Lag model with Reynolds tress tensor implementation \\
905 & lagRST $-\tau_{i j}$ using k- $\omega$ for equilibrium values \\
908 & SST model with Reynolds tress tensor implementation \\
909 & lagRSTSST $-\tau_{i j}$ using SST for equilibrium values \\
\hline
\end{tabular}

The four test cases chosen were the incompressible flat plate of Osterlund ${ }^{30}$ and Osterlund et al., ${ }^{31}$ adverse pressure gradient boundary layer of Driver, ${ }^{11}$ incompressible mixing layer of Bell and Mehta, ${ }^{10}$ and a transonic bump flow by Bachalo and Johnson. ${ }^{12}$ The comparisons shown in the following sections are part of a bigger study done by Lillard. ${ }^{18}$ In particular, many results showing verification of code implementation, detailed grid convergence for multiple models, and velocity and Reynolds stress profile comparisons will not be shown here.

\section{II.A. Incompressible Flat Plate}

Osterlund $^{30}$ and Osterlund et al. ${ }^{31}$ obtained zero pressure gradient turbulent boundary layer data in the Minimum Turbulence Level or Marten Theodore Landahl (MTL) wind-tunnel at the Department of Mechanics, Royal Institute of Technology(Swedish: Kungliga Tekniska Hogskolan, abbreviated KTH). The data was 
taken on a 7 meter flat plate, with instrumentation locations ranging from 1.5 to 5.5 meters. $R e_{\theta}$ ranged from 2500 to 27000 with freestream velocities ranging from 10 to $50 \mathrm{~m} / \mathrm{s}$. Fluctuating velocity components and the fluctuating wall-shear stress were measured. Velocity data was taken with hot-wire anemometry probes mounted on a traversing system that protruded from the plate Skin friction was obtain by using both oil-film interferometry and using the mean velocity measurements and fitting them to the law of the wall with a least squares fit. This test case was first chosen to verify the following:

1. The lagRST model's ability to predict the near wall portion of the turbulent boundary layer (law of the wall).

2. The model's ability to predict the wake portion of the turbulent boundary layer.

To compare velocity profiles, all the models were independently shifted so they matched the experimental value of $\theta$ at $\mathrm{x}=3.5$ meters. This was done by changing the upstream running length of the grid (for each turbulence model) so that at $\mathrm{x}=3.5$ meters the predictions gave the experimental value of $\theta$. This was necessary to remove transition effects from the model comparisons. The velocity profiles were then compared at $\mathrm{x}=5.5$ meters in the shifted coordinate space.

\section{II.A.1. Grid Convergence}

The grids generated were cartesian using geometric spacing in the off-body direction. The nominal grid chosen (referred to as the medium grid) had 92 streamwise points and 129 points in the boundary layer. The grid was extended well beyond the boundary layer edge to remove any boundary consdition effects. The wall spacing chosen gave a $y^{+}$less than 0.15 across the plate. To create the coarse and fine grids, both grid dimensions were scaled by $\sqrt{2}$ to increase the total grid dimension by a factor of 2 . The wall spacing was then changed proportionally. This gave grids with dimensions of 65 by 91 and 129 by 182 .

Figure 1 shows the worst case for grid convergence for the lagRST model, with $\sigma_{k}=0.5$. A non-physical inflection point near the edge of the boundary layer develops for this model. The inflection point begins around $z=0.04 m$. Not only is there a large deviation at the edge, but the non-physical behavior gets worse as the grid density progresses from coarse to fine. This behavior nearly goes away with $\sigma_{k}=0.8$ and completely disappears with $\sigma_{k}=1.5$. Table II.A.1 presents the skin friction values near the end of the flat plate, $\operatorname{Re}_{\theta}=25,000$, for the three variations in $\sigma_{k}$. The $\%$ difference listed is the difference for either the coarse or medium grid from the fine grid solution. $\sigma_{k}=0.5$ clearly has the largest $\%$ differences, indicating a lack of grid convergence to go along with the non-physical velocity profile.

\begin{tabular}{|c|c|c|c|}
\hline Grid & $\sigma_{k}=1.5$ & $\sigma_{k}=0.8$ & $\sigma_{k}=0.5$ \\
\hline coarse & $0.002303(0.35 \%)$ & $0.002263(1.57 \%)$ & $0.02208(2.75 \%)$ \\
\hline medium & $0.002300(0.22 \%)$ & $0.002246(0.81 \%)$ & $0.002176(1.25 \%)$ \\
\hline fine & 0.02295 & 0.002228 & 0.002149 \\
\hline
\end{tabular}

Table 3. Skin friction values at $R e_{\theta}=\mathbf{2 5 0 0 0}$ for the lagRST model with $a_{o}=0.35$ and varying $\sigma_{k}$ with the \% difference between the coarse or medium grid and the fine grid listed in parenthesis.

\section{II.A.2. lagRST Constant Selection}

This section assess the six combinations of $a_{o}$ and $\sigma_{k}$ and removes any pair that generates non-physical behavior. Figure 2 shows the lagRST model with varying coefficients compared to Osterlund's velocity profile data. As it was seen before with previous plots, low values of $a_{o}$ and $\sigma_{k}$ cause an inflection in the boundary layer profile that is not physical. The profiles that retain a shape without an inflection are $a_{o}=0.35$ along with $\sigma_{k}=0.8$ and both solutions with $\sigma_{k}=1.5$. Although clearly not an exact match the the experimental data, the pair of $a_{o}=0.35$ and $\sigma_{k}=0.8$ provides a good representation without having the inflection point the lower values values are plagued with.

Based on the previous figure, several combinations of the constants can be discarded. The inflection in the velocity profiles removes $\sigma_{k}$ values of 0.5 and low $a_{o}$ values with $\sigma_{k}=0.8$. The skin friction comparisons reinforce the removal of $a_{o}=0.2$ with $\sigma_{k}=0.5$ due to how much lower they are than the correlations and data. That leaves three sets of coefficients to assess, $a_{o}=0.35$ with $\sigma_{k}=0.8$ and $a_{o}=0.2$ or $a_{o}=0.35$ with $\sigma_{k}=1.5$. 


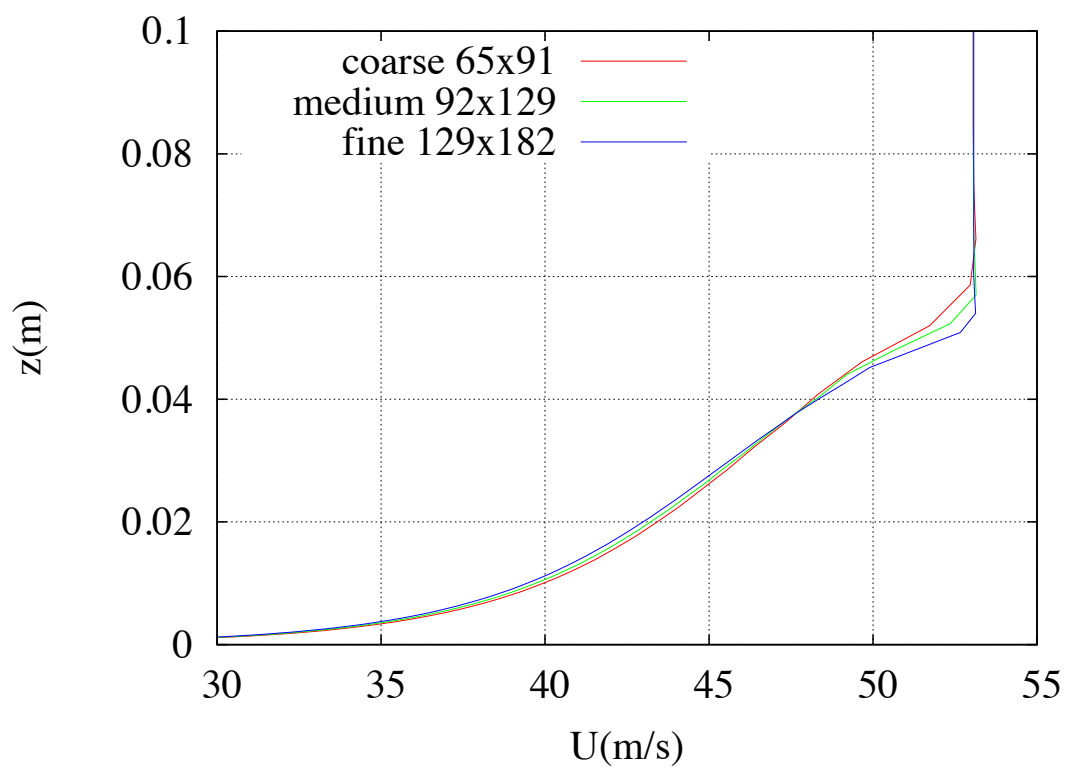

Figure 1. Velocity profiles for lagRST with $\sigma_{k}=0.5$ showing the effects of varying grid dimensions

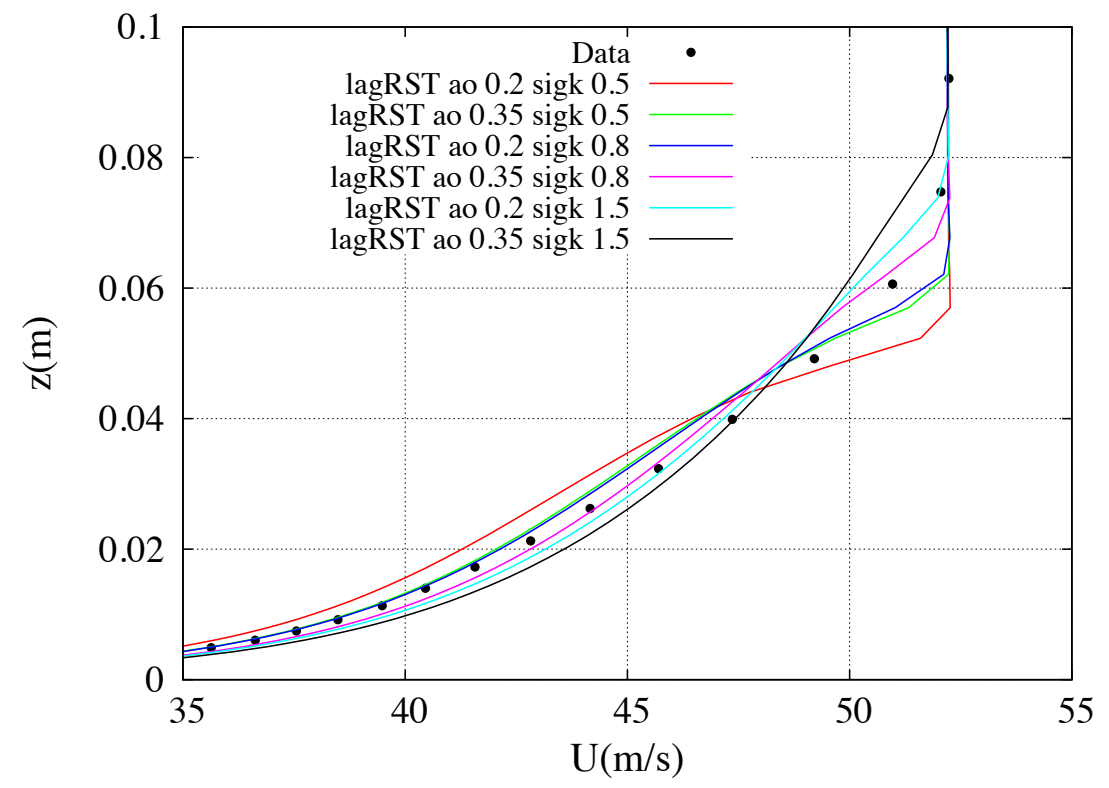

Figure 2. Velocity profiles for the lagRST compared to Osterlund's data.

\section{8 of 37}

American Institute of Aeronautics and Astronautics 


\section{II.A.3. Model Comparisons}

Figure 3 shows the lagRST model with the previously selected combinations of $a_{o}$ and $\sigma_{k}$ as well as the lagRSTSST model compared to the standard SST and SA models. Unfortunately, none of the models match the data across the entire profile. Although none of the models are perfect, it is worth noting that the general error is all on the order of $5-10 \%$. In addition, the inflection near the edge in the lagRST models is avoided based on the selection of constants. For the lagRST models, the two highest coefficients have the most deviation from the experimental data. It can also be said that the edge of the boundary layer the SA model under predicts the velocity magnitude more than the majority of the models.

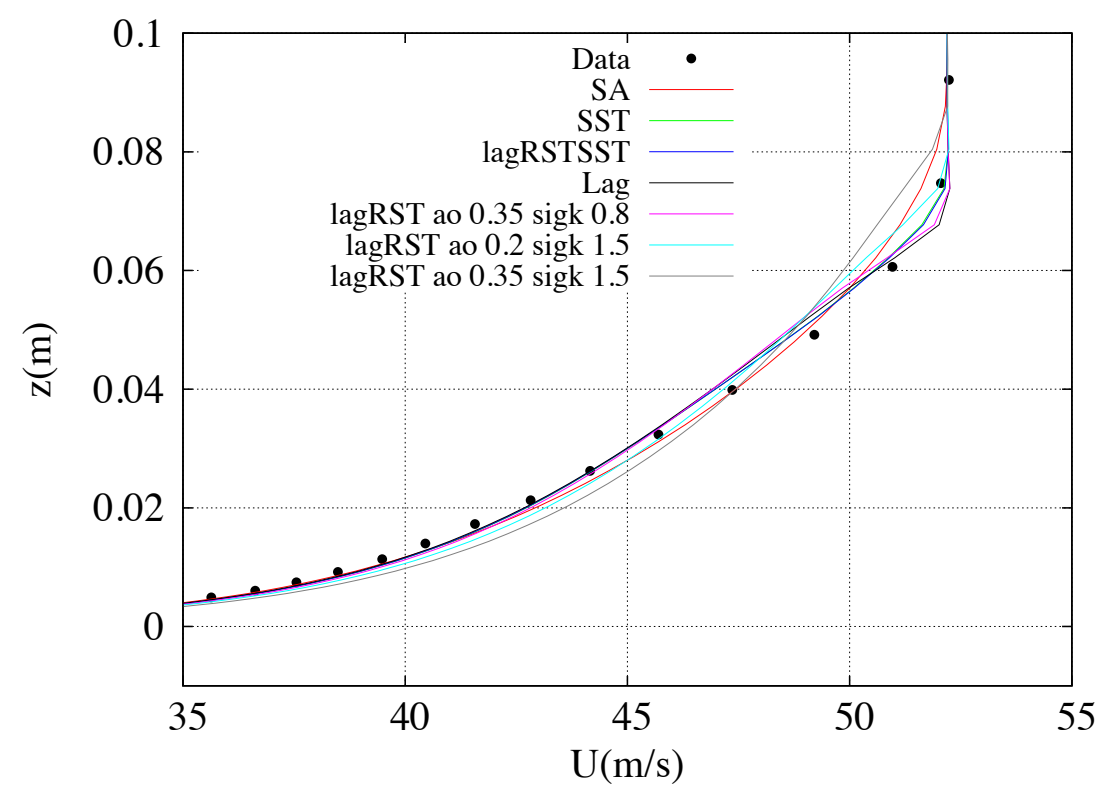

Figure 3. Velocity profiles comparing the Lag, lagRST, lagRSTSST, SST, and SA models

Figure 4 shows the same results in law of the wall space with Coles law of the wake added for comparison. The SA model predicts the transition from the laminar sublayer to the law of the wall region much more accurately than all other models. However, the slope in the law of the wall region is slightly in error compared to the other models. On this scale, the SST models gives a very close result to the Lag and lagRST models with constants $a_{0}$ and $\sigma_{k}$ equal to 0.35 and 0.8 , respectively. None of the models predict the extent and magnitude of the wake region properly.

Skin friction predictions for all the models were within $2 \%$ of each other and are therefore not shown here.

\section{II.B. Mixing Layer}

Bell and Mehta ${ }^{10}$ obtained experimental data for a two-stream mixing layer with a velocity ratio of 0.6 in a Mixing Layer Wind Tunnel located in the Fluid Mechanics Laboratory at NASA Ames Research Center. Measurements were taken with a rotatable cross-wire probe held with a three-dimensional traverse. They obtained data for both tripped and untripped boundary layers. For this comparison, only the tripped turbulent data will be used. They defined the shear-layer thickness, $\delta$, using a least-squares fit of the mean data to the error function profile shape:

$$
U^{*}=\frac{1+\frac{2}{\sqrt{\pi}} \int_{0}^{\eta} e^{-t^{2}} \mathrm{~d} t}{2}
$$

where $\eta$ is defined as 


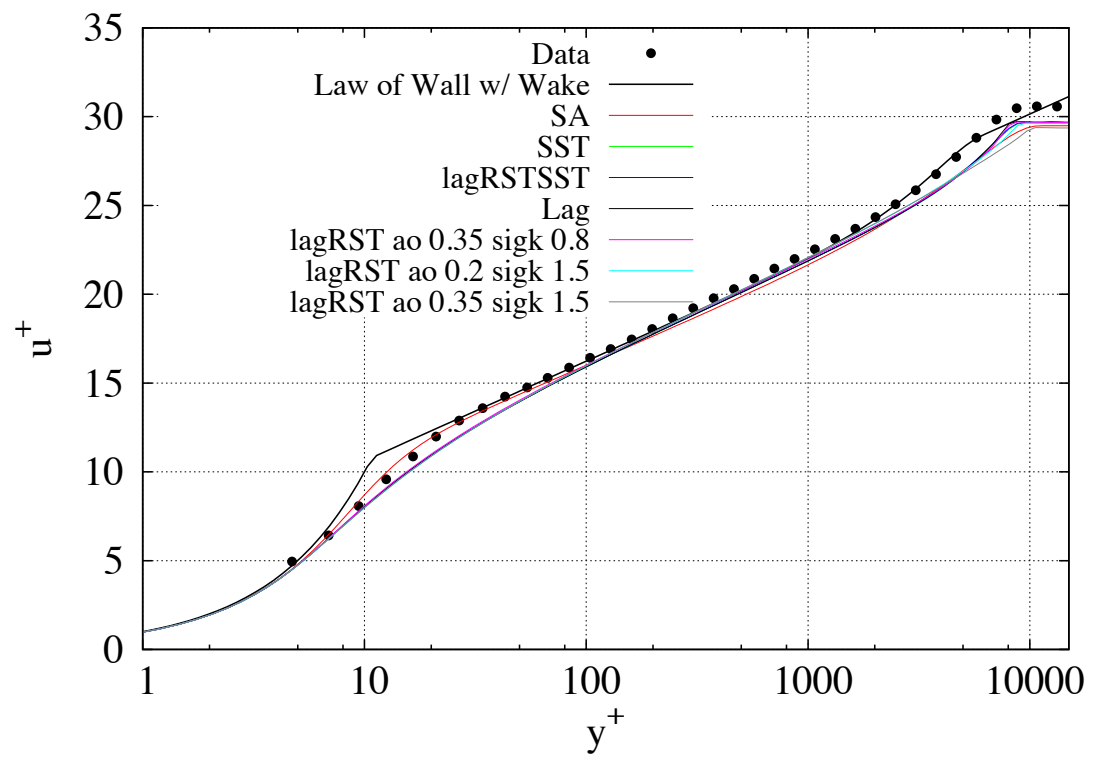

Figure 4. Velocity profile in law of the wall space comparing the Lag, lagRST, lagRSTSST, SST, and SA models with the addition of Cole's law of the wake.

$$
\eta=\frac{Y-Y_{o}}{\delta}
$$

and $U^{*}$, the velocity parameter, is defined as

$$
U^{*}=\frac{U-U_{2}}{U_{1}-U_{2}}
$$

and $Y_{o}$ is the centerline of the mixing layer and is defined from the error function fit. The average RMS error for the least squares fit had less than a $2 \%$ deviation between the functional form and the data. To compare to this data, the velocity profiles were extracted and then processed through a least squares fit to calculate $\delta$ and $Y_{o}$. The upper velocity stream, $U_{\text {upper }}$, was set to $15 \mathrm{~m} / \mathrm{s}$ while the lower velocity stream, $U_{\text {lower }}$, was set to $9.0 \mathrm{~m} / \mathrm{s}$. The solution was initialized for all positive $\mathrm{z}$ values to $U_{\text {upper }}$ and negative $\mathrm{z}$ values to $U_{\text {lower. }}$. The inflow condition was set to the same velocity condition as the solution initialization and used an inflow/outflow condition. The upper and lower conditions were set to $U_{\text {upper }}$ and $U_{\text {lower }}$, respectively and used the same inflow/outflow condition as the initial condition. The downstream boundary condition was set to outflow (extrapolation).

\section{II.B.1. Grid Convergence}

Three grids were generated in order to study grid convergence for this test case. The finest grid ran was 129 (axial) by 205. The grid was then decreased to 65 by 103 and further to 33 by 52 . Figures 5 shows grid convergence for the lagRST and lagRSTSST models. For the lagRSTSST model, the medium grid (65 by 103) gets within $2 \%$ of the fine grid answer. For the lagRST again the medium grid gives excellent grid convergence and is within $1 \%$.

\section{II.B.2. Model Comparisons}

Figure 6 shows a comparison of the SST, lag, lagRST, and lagRSTSST models. The lagRSTSST compares very well with the standard two-equation SST model and the experimental data. The lagRST and Lag model comparisons are very poor, even with large variations in $\sigma_{k}$. To date, there has been no explanation for this. 


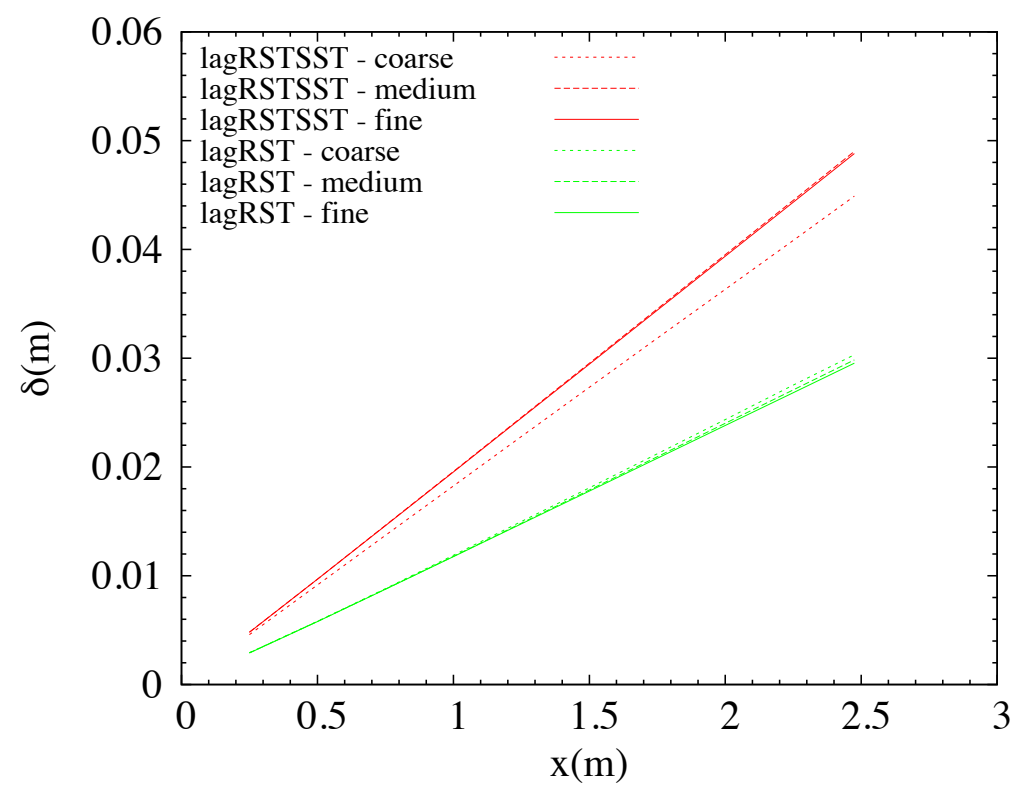

Figure 5. Grid convergence for the lagRST and lagRSTSST models compared to the mixing layer growth data for the fine (129 by 205), medium (65 by 103), and coarse (33 by 52) grids.

For the lagRSTSST model, a lower value of the lag constant, $a_{o}=0.2$, was assessed for this case. It had little effect, but did increase the slope of the growth rate by $1.5 \%$.

\section{II.C. Driver's CS0 Flowfield}

Driver $^{11}$ executed an incompressible test of a turbulent separated boundary layer in a low speed open circuit wind tunnel. The test geometry was a circular cylinder aligned with the freestream and mounted in the center of the tunnel. It was surrounded by flexible tunnel walls that could be diverged in order to create an adverse pressure gradient. Skin friction measurements were taken with an oil-flow laser interferometer. Profile data was taken with a three-component laser Doppler velocimeter (LDV) system.

The computational geometry was modeled with an axisymmetric cylinder to define the surface and an external streamline taken from the experimental data to define the opposite boundary. The outer streamline was treated as an inviscid wall. The viscous wall is no-slip and adiabatic. The upstream boundary condition was defined as constant total pressure and temperature, with static pressure allowed to vary and velocity direction aligned with the cylinder axis. The downstream static pressure was adjusted at the exit to match the experimental static pressure upstream of the interaction region $(x=-0.438 m)$. This was an iterative process that was done for each simulation. In addition, the upstream length was also taken from Olsen and Coakley $^{7}$ to match the experimental boundary layer thickness.

The calculations were made on the same grids used in the original analysis by Olsen and Coakley. ${ }^{7}$ The fine grid had 200 (axial) by 160 (radial) points. The coarse grid had a factor of 2 reduction in grid points (100 by 80 ) and the medium grid had 150 by 120 . Olsen used the grids referred to as fine and coarse in his study. Figure 7 shows the computational domain for this test case.

\section{II.C.1. Grid Convergence}

Figure ?? shows the skin friction distribution for the lagRSTSST model. The model has excellent convergence behavior. There are mild differences well upstream, but once the adverse pressure gradient area starts all models are in agreement. The pressure comparisons were nearly identical between the grids, thus they are not pictured here.

The medium and fine grids for all cases matched the measured upstream boundary layer thickness to within $10 \%$. 


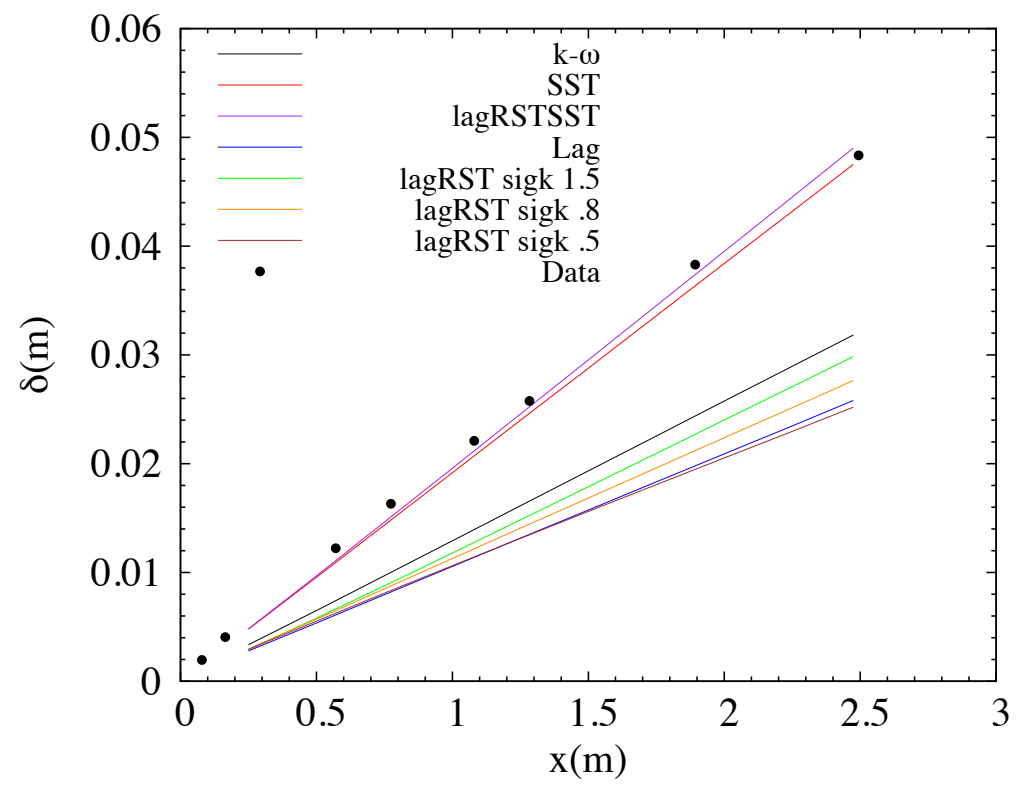

Figure 6. Mixing layer growth rate comparisons for the SST, lag, lagRST, and lagRSTSST models.

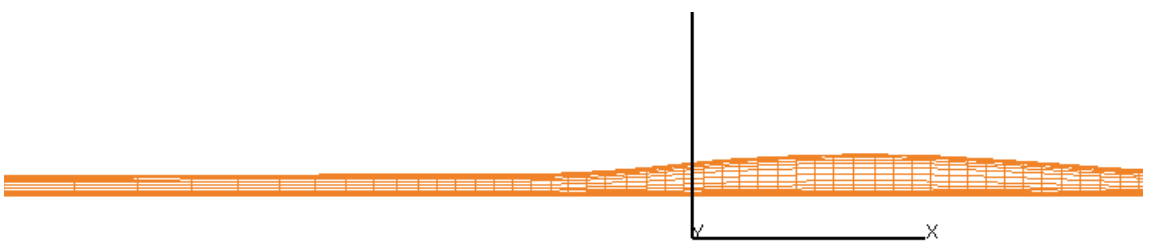

Figure 7. Computational domain, coarse grid pictured. 


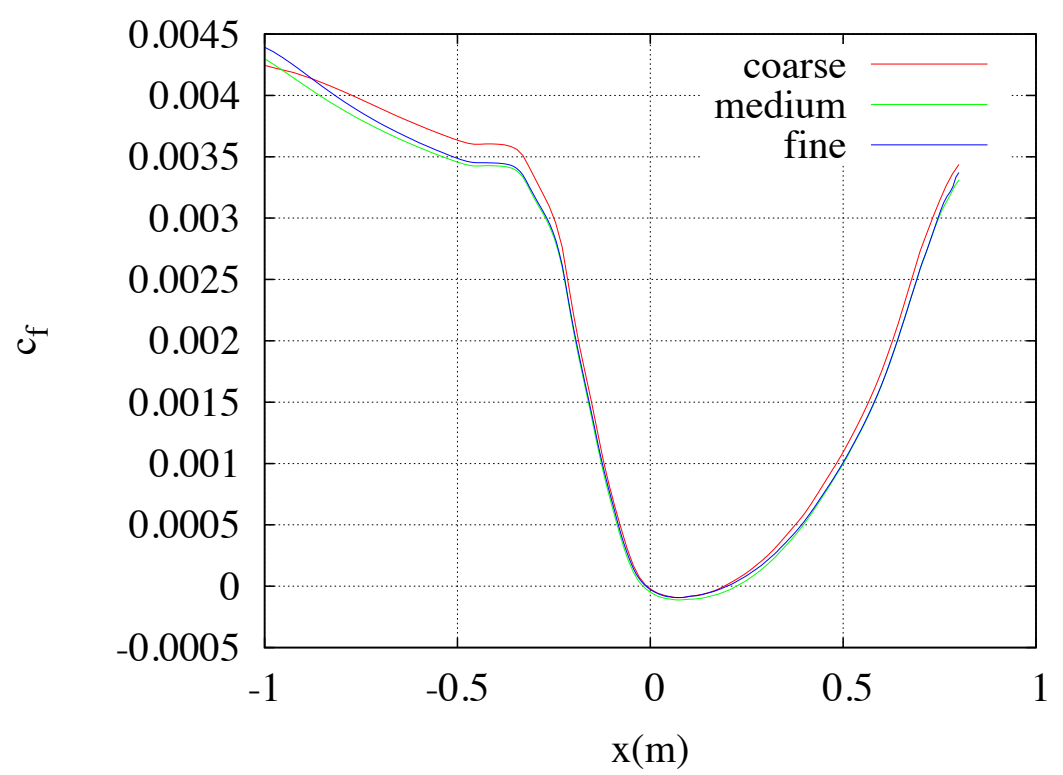

Figure 8. Skin friction comparisons showing grid convergence for the lagRST model with standard coefficients.

Although now shown here, the lagRST model gave comparable results. The medium and fine grids for all cases matched the measured upstream boundary layer thickness to within ten percent. It was also confirmed that the grid system had acceptable convergence performance when looking at the Reynolds stresses (although not shown here).

\section{II.C.2. Model Comparisons}

In previous sections, the lagRST coefficients have been reduced to three sets of choices. For ease of reference, table II.C.2 shows the new naming convention for the lagRST based models for the three different sets of coefficients.

Table 4. Model naming convention for lagRST models

\begin{tabular}{|c|c|c|}
\hline lagRST model name & $\sigma_{k}$ & $a_{o}$ \\
\hline lagRST-1 & 0.8 & 0.35 \\
lagRST-2 & 1.5 & 0.2 \\
lagRST-3 & 1.5 & 0.35 \\
\hline
\end{tabular}

Figure 9 shows the pressure distribution versus axial distance for the SST, Lag, lagRST, and lagRSTSST models. For the lagRST models, the lagRST-2 model matches the pressure more accurately. However, all the lagRST models are an improvement over the baseline $k-\omega$ model. The Lag and lagRST-2 provide the best prediction for these models. The SST-based models provide the best overall prediction of pressure. The lagRSTSST model predicts the pressure to have a lower plateau in the interaction region than the SST model. Overall, adding the lag equation improves the predictions for the baseline models. The lagRSTSST model was assessed with a lag constant of $a_{o}=0.2$ for this case. It had less than a $1 \%$ change on the size and location of the separation zone vs the lagRSTSST model with the standard coefficient.

Figures 10 and 11 detail the skin friction distribution on the cylinder. There is insufficient data to exactly characterize the separation zone length using skin friction, so the experimental separation length was determined with oil flow interferometry. The separation zone ranged from approximately from $x=30 \mathrm{~mm}$ to $x=220 \mathrm{~mm}$. This was within $\pm 25 \mathrm{~mm}$ of the mean flow data. Table II.C.2 lists the separation and reattachment points as well as separation length for all models. 


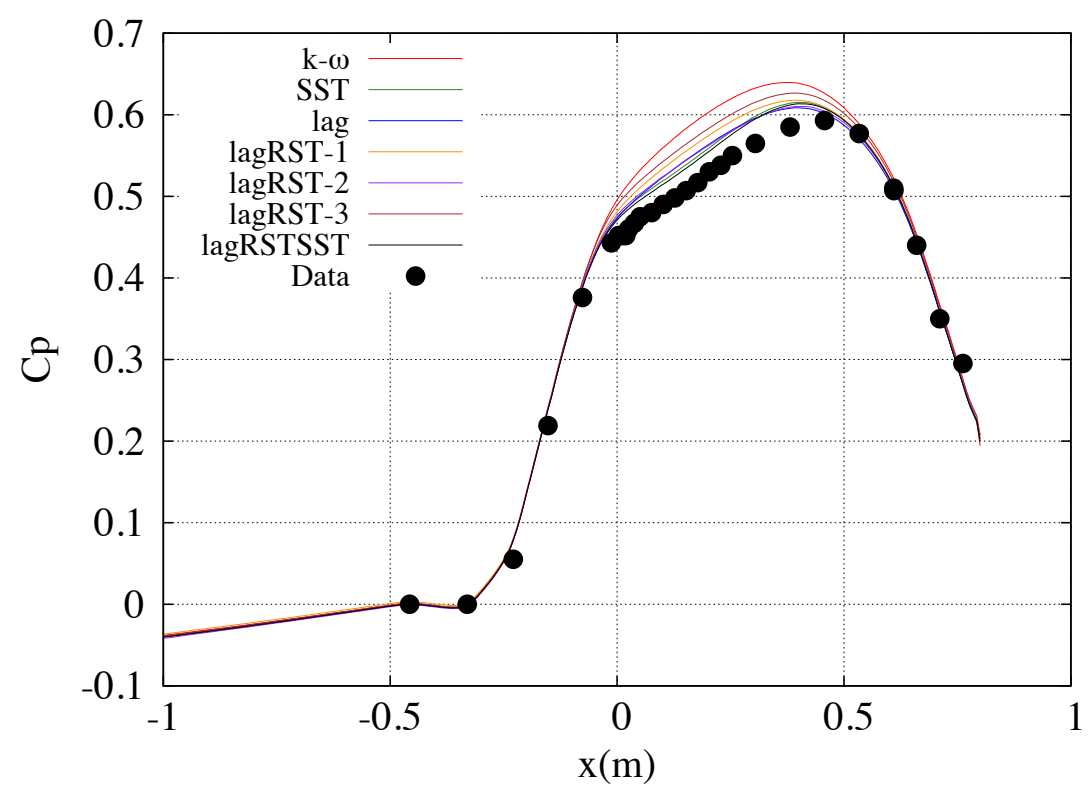

Figure 9. Pressure versus streamwise locations for the Lag, lagRST, and lagRSTSST models compared to $k-\omega$ and SST.

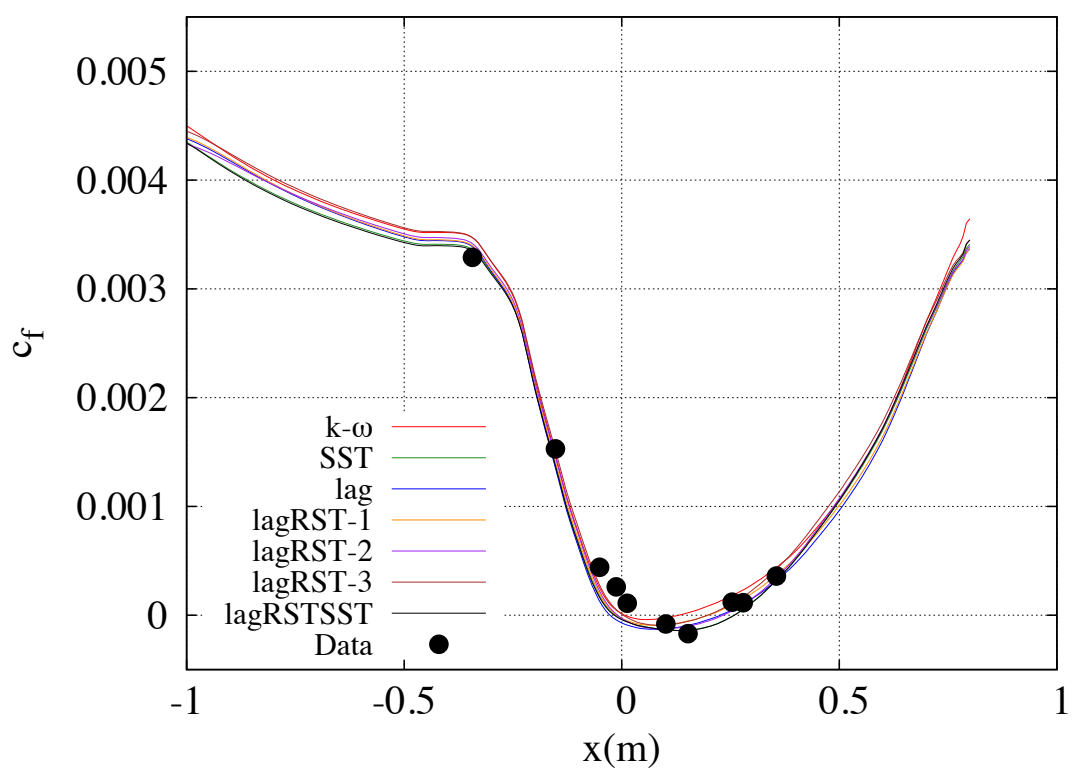

Figure 10. Skin friction versus streamwise locations for the Lag, lagRST, and lagRSTSST models compared to $k-\omega$ and SST, with the separation noted by arrows. 


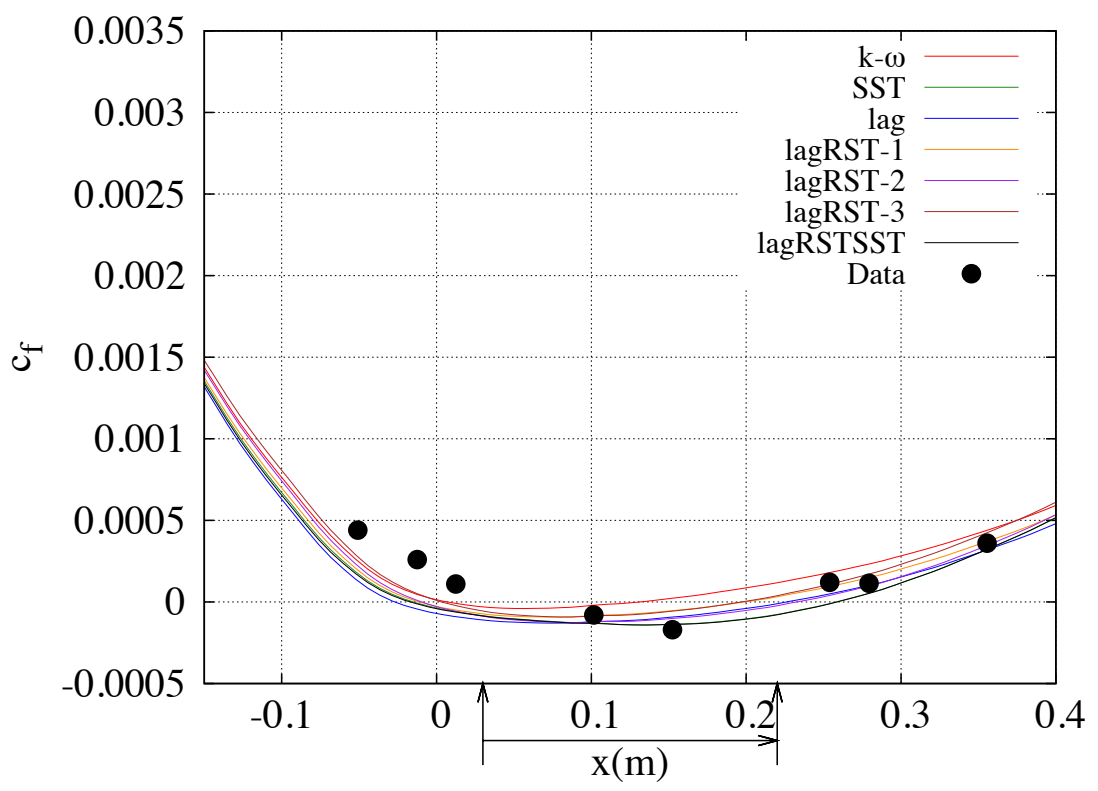

Figure 11. Skin friction versus streamwise locations for the Lag, lagRST, and lagRSTSST models compared to $k-\omega$ and SST focusing on the separation region.

All the $k-\omega$ based RST models provide a more accurate separation zone length prediction than the SST-based models. By far the best comparison comes form the lagRST model with $a_{0}=0.35$ with either value of $\sigma_{k}$ chosen (lagRST-1 or lagRST-3). It is interesting to note that the baseline $k-\omega$ model is the only one which predicts a smaller separation zone length than the experiment. The lagRSTSST model provides the worst separation zone length prediction of any model. The majority of the models do a better job characterizing the reattachment point than the separation point. None of the models provide a prediction of the separation point that is within $12 \%$ of the experimental results, whereas two of the models provide reattachment predictions within $12 \%$ of the experimental results.

Table 5. Table of data indicating location and extent of the separation zone with percent error compared to the tunnel data. The percent error uses the separation zone length in the denominator.

\begin{tabular}{|c|c|c|c|}
\hline Model & Separation Point & Reattachment & Zone length \\
\hline Data & $0.03 \mathrm{~m} \pm 0.025$ & $0.22 \mathrm{~m} \pm 0.025$ & $0.19 \mathrm{~m} \pm 0.025$ \\
\hline $\mathrm{k}-\omega$ & $0.00708(-12.1 \%)$ & $0.131(-46.6 \%)$ & $0.124(-34.5 \%)$ \\
SST & $-0.015(-23.6 \%)$ & $0.258(20.0 \%)$ & $0.273(43.6 \%)$ \\
Lag & $-0.0269(-29.9 \%)$ & $0.226(3.16 \%)$ & $0.253(33.1 \%)$ \\
lagRST-1 & $-0.011(-21.6 \%)$ & $0.199(-11.2 \%)$ & $0.210(10.4 \%)$ \\
lagRST-2 & $-0.00955(-20.8 \%)$ & $0.233(6.67 \%)$ & $0.242(27.5 \%)$ \\
lagRST-3 & $0.00328(-14.1 \%)$ & $0.197(-12.2 \%)$ & $0.194(1.8 \%)$ \\
lagRSTSST & $-0.0168(-24.6 \%)$ & $0.259(20.4 \%)$ & $0.276(45.0 \%)$ \\
\hline
\end{tabular}

\section{II.D. Transonic Bump Flow}

Bachalo and Johnson ${ }^{12}$ tested a transonic interaction on an axisymmetric cylinder with a circular arc bump. This test was executed in the NASA Ames Research Center $2 \times 2$ foot Transonic Wind Tunnel. Data was taken with a two velocity-component laser velocimeter. The upstream boundary conditions were constant total pressure and temperature, with static pressure allowed to vary and velocity direction aligned with the cylinder axis. The outer edge of the flowfield was extended approximately 10 bump cord lengths away from the cylinder surface and utilized a characteristic boundary condition. The viscous wall was no-slip adiabatic 
with the downstream static pressure held at $p_{\text {inf }}$. To maintain consistency with Olsen and Coakley, ${ }^{7}$ the upstream length of the cylinder was maintained at the value they used, which provided a boundary layer thickness of approximately $1 \mathrm{~cm}$ at the location of the bump, which was the same approximate thickness measured in the test.

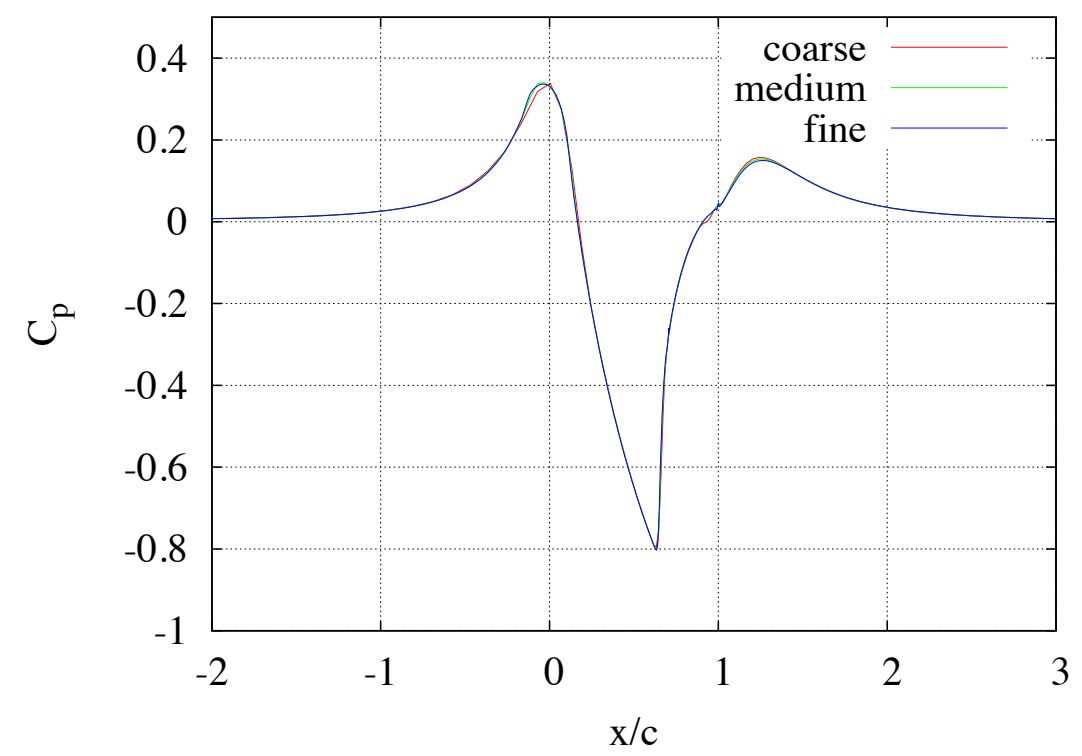

Figure 12. Pressure plots for the lagRST-1 model showing grid convergence.

\section{II.D.1. Grid Convergence}

Three grids were chosen for this case. A coarse grid with dimensions of 96 (axial) by 101 (off-body), medium grid with dimensions 192 by 151, and a fine grid with dimensions 390 by 201. Wall spacings that translated to $y^{+}$values less than $0.2,0.1$, and 0.05 were used for the coarse, medium, and fine grids, respectively.

Figure 12 shows the pressure comparisons for the lagRST-1 model for the various grid resolution cases. The medium and fine grid give nearly identical results. The other models (lagRST-2, lagRST-3, and lagRSTSST), all had similar convergence behavior, if not better. The medium grid provides adequate grid convergence for this test case.

\section{II.D.2. Model Comparisons}

Figure 13 shows the pressure comparisons for the lagRST based models as well as the k- $\omega$, SST, and Lag models focused in on the the interaction region. The prediction of the shock location is of vital importance, but is not consistently predicted by the models. The $\mathrm{k}-\omega$ predicts a very late shock location and the lagRSTSST predicts it very early. These two models bracket the other models. The SST and the lagRST-2 models give nearly identical answers. They predict a slightly upstream location for the shock. The Lag and lagRST-1 also give nearly identical answers to each other, and provide a very accurate prediction for the shock location. The lagRST-3 also gives a very accurate shock location. None of the models predict the right pressure behavior in the separation zone $(x=0.71 m-1.1 m)$. The lagRSTSST model has the closest comparison. However, none of the models predict the steep drop-off in pressure just after re-attachment that is present in the experimental data.

Besides shock location and pressure comparisons, perhaps the more important comparison to make is of the separation zone length. Table II.D.2 shows the observed separation and attachment points as well as separation zone lengths for each turbulence model. The table indicates the $\mathrm{k}-\omega$ model provides an accurate prediction of the separation zone, but this only refers to the extent of the separation. Previous figures have 


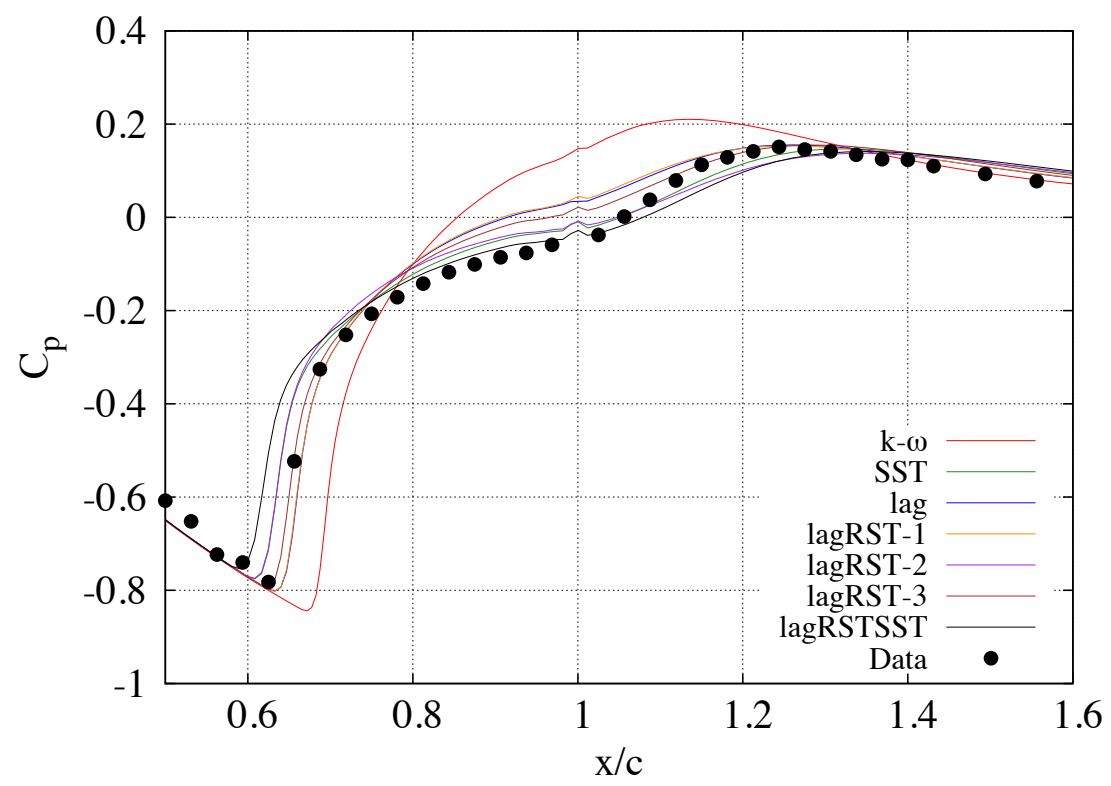

Figure 13. $C_{p}$ versus axial location for all turbulence models compared to the experimental data focusing on the separation zone.

shown that the pressure prediction in the separation zone from this model is under predicted. The Lag and lagRST-1 models provide the best characterization of the separation zone length, with the worst comparisons coming from the SST and the lagRSTSST models. The lagRST-3 and lagRST-2 models (which both use a larger value of $\sigma_{k}$ ), also have a large error in the prediction. The lagRSTSST model was assessed with a lag constant value of $a_{o}=0.2$. This had no effect on separation and less than $0.1 \%$ change on the separation zone length and position.

Table 6. Table of data indicating location and extent of the separation zone with percent error compared to the tunnel data. The percent error uses the separation zone length as the denominator.

\begin{tabular}{|c|c|c|c|}
\hline Model & Separation Point & Reattachment & Zone length \\
\hline Data & 0.71 & 1.1 & 0.39 \\
\hline k- $\omega$ & $0.701(-3.03 \%)$ & $1.05(-13.8 \%)$ & $0.346(-10.8 \%)$ \\
SST & $0.645(-17.4 \%)$ & $1.18(21.6 \%)$ & $0.539(39.0 \%)$ \\
Lag & $0.666(-11.9 \%)$ & $1.12(4.64 \%)$ & $0.452(16.6 \%)$ \\
lagRST-1 & $0.668(-11.4 \%)$ & $1.12(4.14 \%)$ & $0.210(10.4 \%)$ \\
lagRST-2 & $0.646(-17.1 \%)$ & $1.19(21.9 \%)$ & $0.539(39.1 \%)$ \\
lagRST-3 & $0.659(-13.7 \%)$ & $1.15(12.5 \%)$ & $0.5(26.3 \%)$ \\
lagRSTSST & $0.632(-20.8 \%)$ & $1.19(23.3 \%)$ & $0.556(44.1 \%)$ \\
\hline
\end{tabular}

\section{II.E. Shock Impinging on a Turbulent Boundary Layer}

Schulein ${ }^{32,33}$ conducted a $\left(M_{\infty}=5\right)$ wind tunnel test in the DLR Gottingen Ludwieg Tube facility, using air as the test gas. The nominal freestream conditions for this test are listed in table 7 . The measured values included wall pressure, skin friction (by an oil-flow technique), and wall heat transfer (infrared camera measurements and thin film gages). The provided experimental uncertainties were approximately $2 \%$ for pressure, $10 \%$ for skin friction, and $20 \%$ for heat flux. The upstream boundary layer was fully developed and an assessment by Brown ${ }^{34}$ showed that it adhered to standard turbulent correlations.

Schulein tested four configurations, starting from a 2-D nominal zero-pressure gradient flat plate boundary layer and then adding a shock generator with 3 different angles, $6^{\circ}, 10^{\circ}$, and $14^{\circ}$. Brown ${ }^{34}$ did an exhaustive 
Table 7. Nominal test conditions for Schulein's experiment.

\begin{tabular}{|c|c|}
\hline Condition & Value \\
\hline$P_{T}$ & $2.12 \mathrm{MPa}$ \\
$T_{0}$ & $410 \mathrm{~K}$ \\
$T_{w}$ & $300 \mathrm{~K}$ \\
$R e / m$ & $36 x 10^{6}$ \\
$U_{\infty}$ & $830 \frac{m}{s}$ \\
$H_{0, \infty}$ & $0.41 \frac{M J}{k g}$ \\
\hline
\end{tabular}

study of all three angles and provided recommendations for which cases to run. Other comparisons to this dataset are available in the literature, including Fedorova et al. ${ }^{35}$ and Steelant. ${ }^{36}$ For this study, the 2-D boundary layer was assessed to ensure the quality of the numerical upstream profile before the interaction. The only shock generator angle assessed was the $14^{\circ}$ case. This case has the largest separation zone tested by Schulein. In addition, it has been chosen by NASA's Fundamental Aeronautics Program as a major test case to use for model comparison. The lower shock generator angles have attached or incipient separation, thus do not assess the separation behavior of the model.

An issue that plagues most cases that have this configuration is the shock generator is not relatively "long" compared to the nominal boundary layer thickness. This allows the expansion waves that emanate from the tail of the shock generator to interfere with the separation region, making the interaction more complex and not allowing for the various topological features of the SWTBLI flowfield to develop. Schulein was able to have a $\frac{L_{g e n}}{\delta_{0}} \approx 1000$, which is a large enough ratio to provide ample space for the interactions to set up.

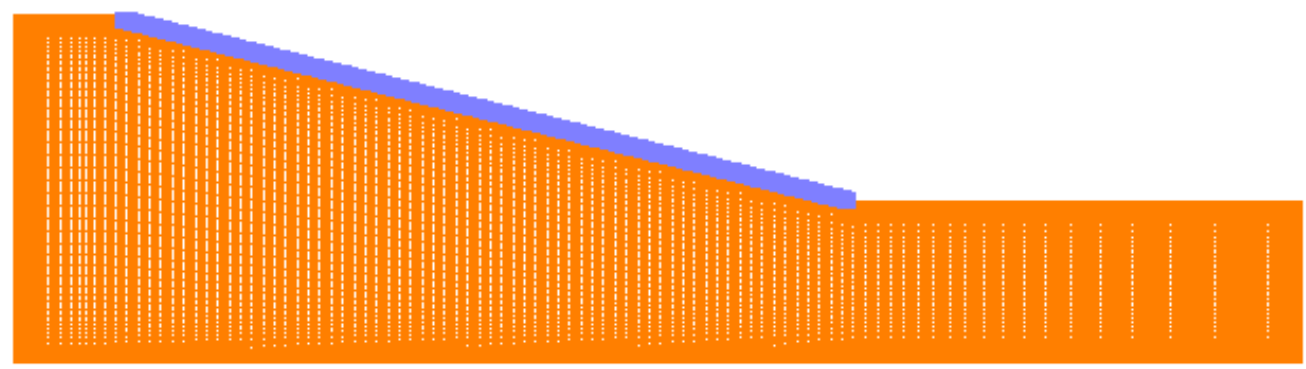

Figure 14. Coarse grid for the $14^{\circ}$ SWTBLI case (blue line indicates the shock generator).

\section{II.E.1. Grid System}

The initial grid system was identical to the set run by Brown. ${ }^{34}$ Table 8 gives the dimensions and $y^{+}$values for each grid system provided by Brown. Figure 14 shows the grid for the $14^{\circ}$ SWTBLI, where flow would be from left to right. The blue line indicates the viscous wall of the shock wave generator. The adjacent boundary conditions to the shock generator were set to inviscid walls, and the opposite wall was set to a viscous, isothermal wall.

Table 8. Grid dimensions and $y^{+}$values for the nominal set of grids.

\begin{tabular}{|c|c|c|c|}
\hline Grid System & streamwise & off-body & $y^{+}$ \\
\hline coarse & 529 & 129 & 0.1 \\
medium & 1057 & 257 & 0.05 \\
fine & 2113 & 513 & 0.025 \\
\hline
\end{tabular}




\section{II.E.2. Flat Plate Boundary Layer Results}

This case followed the standard method used in all other cases to setup the incoming turbulent boundary layer profile. This profile will be generated on the actual geometry by transitioning the simulation in order to match the momentum thickness calculated from the experimental velocity profiles. This is not the method Brown $^{34}$ used, as he numerically set the transition point based on the experimentally observed location. This method did not work in OVERFLOW, as there is no way to control the transition zone length. In particular, each turbulence model behaves differently in this transition zone, which made the results vary based on the model. Therefore, the previous method of setting the transition point to match the experimentally provided $\theta$ was used. This method provides the best estimate of the incoming turbulent boundary layer.

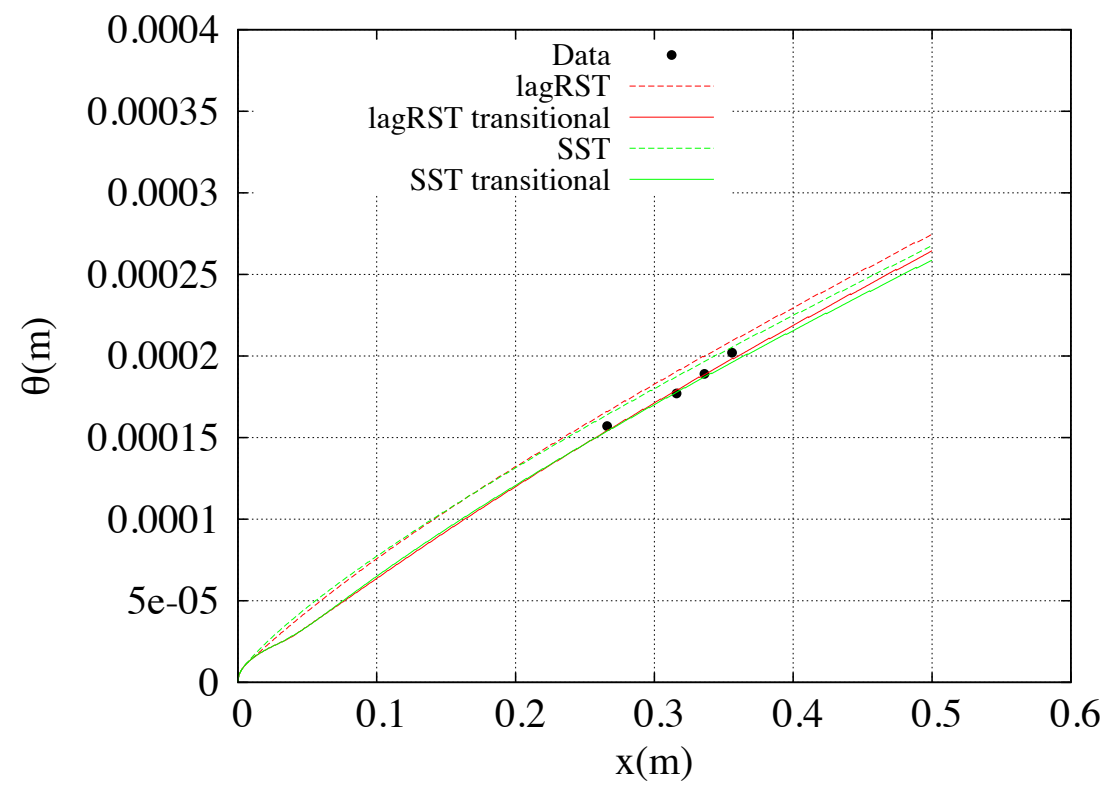

Figure 15. Momentum thickness verses axial distance for the SST and lagRST-1 models compared to Schulein's 2-D boundary layer data.

Figure 15 shows the matching process for the SST and lagRST-1 models. The dashed lines are the fully turbulent cases while the solid lines have been tripped to match $\theta$. Both cases were transitioned at different axial locations. The same process was done for all other turbulence models, but will not be shown here. This process shifts the virtual origin of the simulations so that the boundary layers between different simulations and the experimental data are in the same reference frame. Figure 16 shows the heat flux for the same profiles. The predictions go through the scatter in the data. This profile shows that once sufficiently downstream, the tripping process makes little difference on the value of heat flux (shown by the small differences between the dashed and solid lines of the same color). The Reynolds number is sufficiently high that downstream of the interaction, the transitional effects on heat flux are minimal.

\section{II.E.3. Grid Convergence}

The baseline set of grids run for this case were provided by Brown. ${ }^{34}$ The dimensions were previously listed in table 8. All grid resolution cases were run fully turbulent to avoid sensitivities to transition location and the extent of the transition zone. Figure 17 shows the pressure distribution for all three grids for the SST model. The medium and fine grid show excellent grid convergence, even for the separation location. The lagRST model did not converge with the medium grid, but further testing showed the fine grid was converged. 


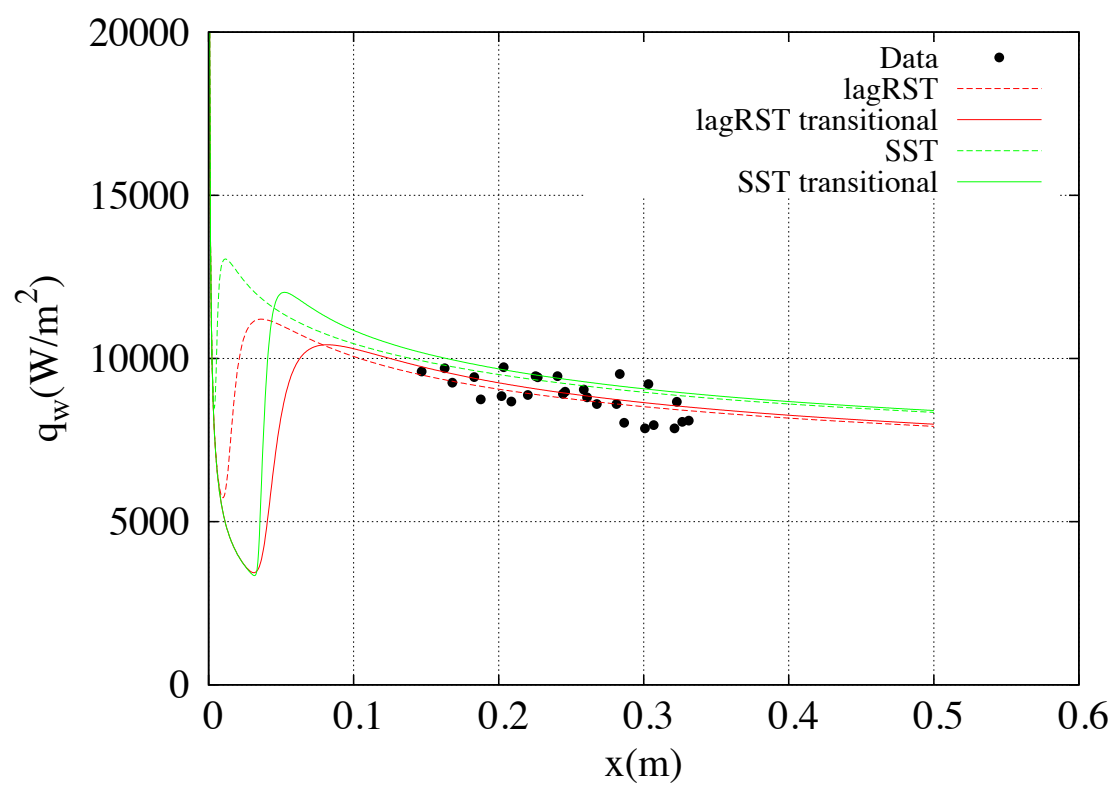

Figure 16. Heat flux $\left(W / m^{2}\right)$ verses axial distance for the sst and lagRST-1 models compared to Schulein's 2-D boundary layer data.

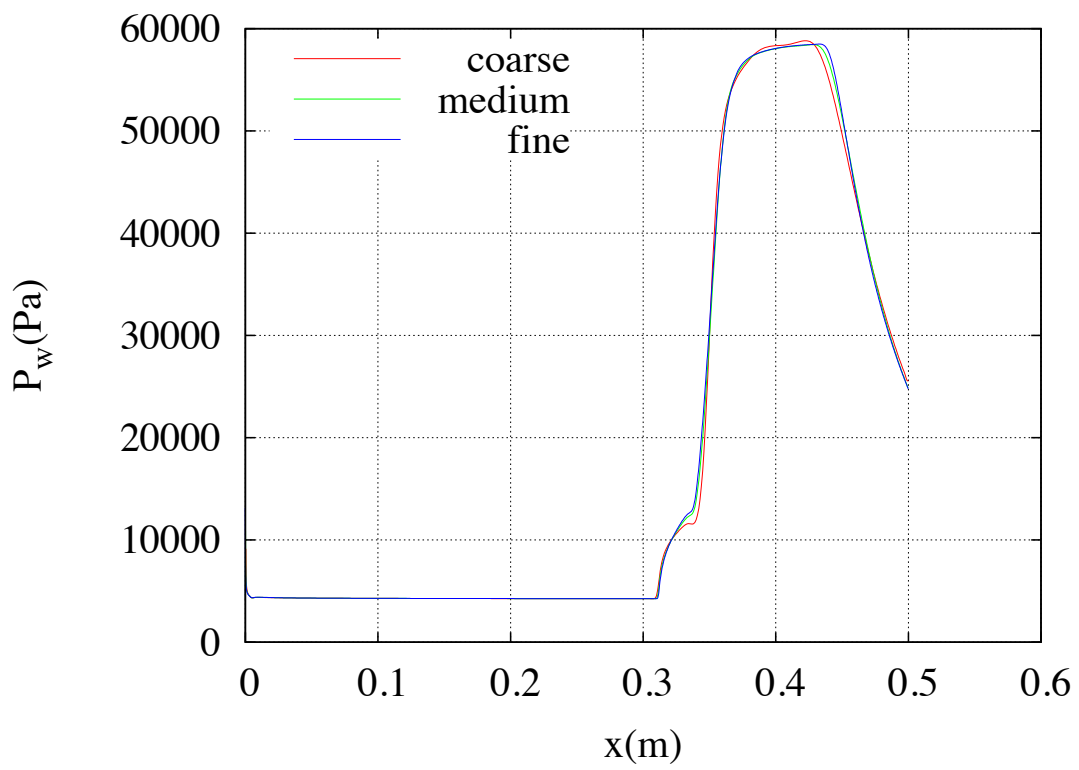

Figure 17. Pressure $(P a)$ distribution for the SST model for the coarse, medium, and fine grid. 


\section{II.E.4. $14^{\circ}$ SWTBLI Data Comparisons}

Table II.E.4 lists the separation and reattachment point along with the separation zone length for each model run. The SST model has by far the best characterization of the separation zone length and location. The percent errors between the simulations and the experiment for the separation zone length are less than $1 \%$. The remaining models do a very poor job predicting the separation point, with the majority of the models having percent errors over $30 \%$. The reattachment point is well characterized by all the models (numerical prediction of reattachment location is within $0.1 \%$ for all the simulations). The separation zone length thus has the same characteristics as the separation point uncertainties. The SST provides an extremely accurate answer and the remaining models have percent errors greater than or equal to $30 \%$. The accuracy of the simulations at reattachment is an interesting result. Previous computations with the Lag model by Oliver et al. ${ }^{37-39}$ on similar SWTBLI (Mach 3 compression ramps) had variations in the prediction of both the separation and re-attachment points. It is not clear why the shock impinging simulations predict the experimentally observed re-attachment point. The re-attachment appears to be driven by the external streamlines being processed through multiple shocks which re-align the inviscid flowfield with the viscous wall. However, this observation is largely geometric in nature, thus the exact physics of this phenomenon are unknown at this time.

Table 9. Table of data indicating location and extent of the separation zone with percent error compared to the tunnel data. The percent error uses the separation zone length as the denominator.

\begin{tabular}{|c|c|c|c|}
\hline Model & Separation Point & Reattachment & Zone length \\
\hline Data & $0.314 \mathrm{~m}$ & $0.348 \mathrm{~m}$ & $0.034 \mathrm{~m}$ \\
\hline SA & $0.327(39.2 \%)$ & $0.349(4.2 \%)$ & $0.0221(-35.0 \%)$ \\
SST & $0.314(0.6 \%)$ & $0.348(0.7 \%)$ & $0.034(0.1 \%)$ \\
Lag & $0.300(42.5 \%)$ & $0.349(3.5 \%)$ & $0.0496(46.0 \%)$ \\
lagRST-1 & $0.296(52.9 \%)$ & $0.351(7.7 \%)$ & $0.0546(60.7 \%)$ \\
lagRST-3 & $0.301(39.5 \%)$ & $0.348(7.0 \%)$ & $0.0498(46.6 \%)$ \\
lagRSTSST & $0.304(29.1 \%)$ & $0.348(0.7 \%)$ & $0.0441(29.8 \%)$ \\
lagRSTSST low $a_{o}$ & $0.295(56.6 \%)$ & $0.35(4.9 \%)$ & $0.0549(61.5 \%)$ \\
\hline
\end{tabular}

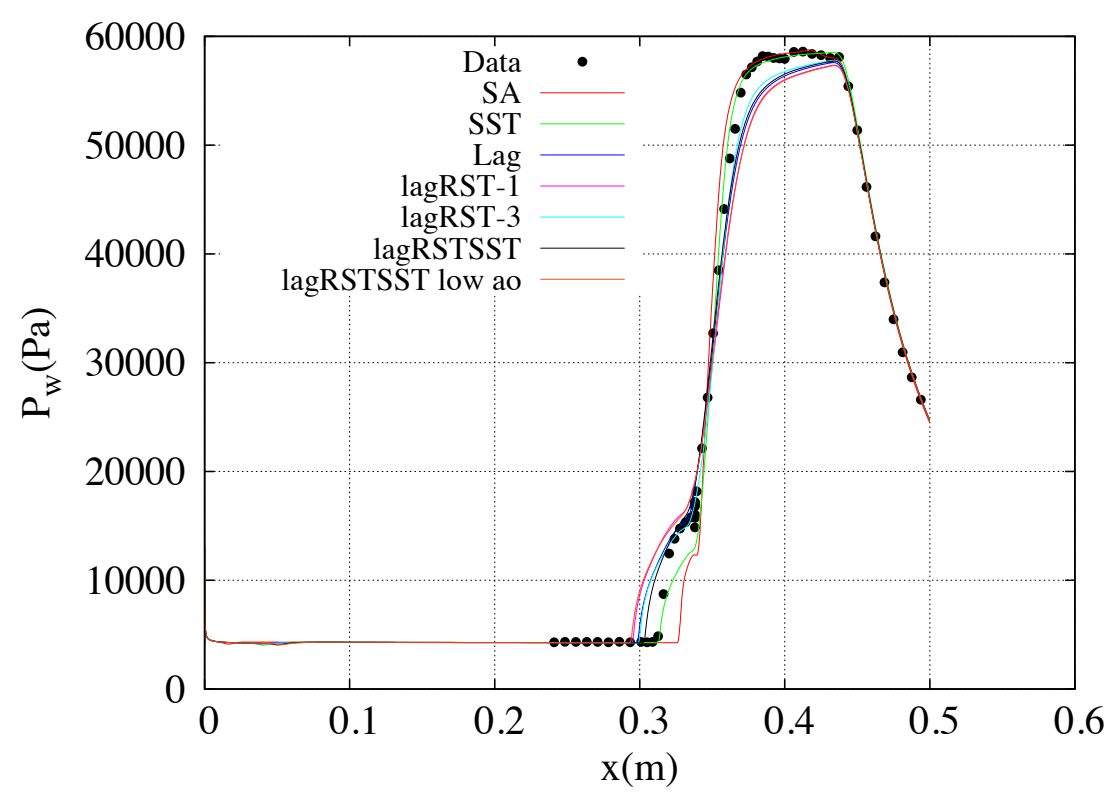

Figure 18. Pressure (Pa) distribution comparisons for all turbulence models on the fine grid. 
Although the SST model matched the separation zone extent nearly perfectly, it does under predict the pressure plateau in the separation zone. Figure 18 compares the wall pressure distribution for all the models. The SA and SST models clearly miss the pressure plateau by around $30 \%$. This is in contrast to the lagRSTSST and the lagRST models which match the pressure plateau very well. The lagRST-1, lagRST-3, and lagRSTSST with the low $a_{o}$ over predict the pressure plateau, but by a small percentage. The under predicted pressure in the separation zone is contradictory to the observation of Wilcox ${ }^{14}$ previously discussed. Wilcox found that the pressure in the separation zone was typically over predicted by turbulence models. The opposite trend in pressure comparisons is true for the pressure peak behind the reflected shock. The SA and SST models predict the peak very well, whereas all the lag-based models under predict the peak across the plateau region until the very end of the region. The SA and SST models predict the very flat pressure profile measured in the wind tunnel data.

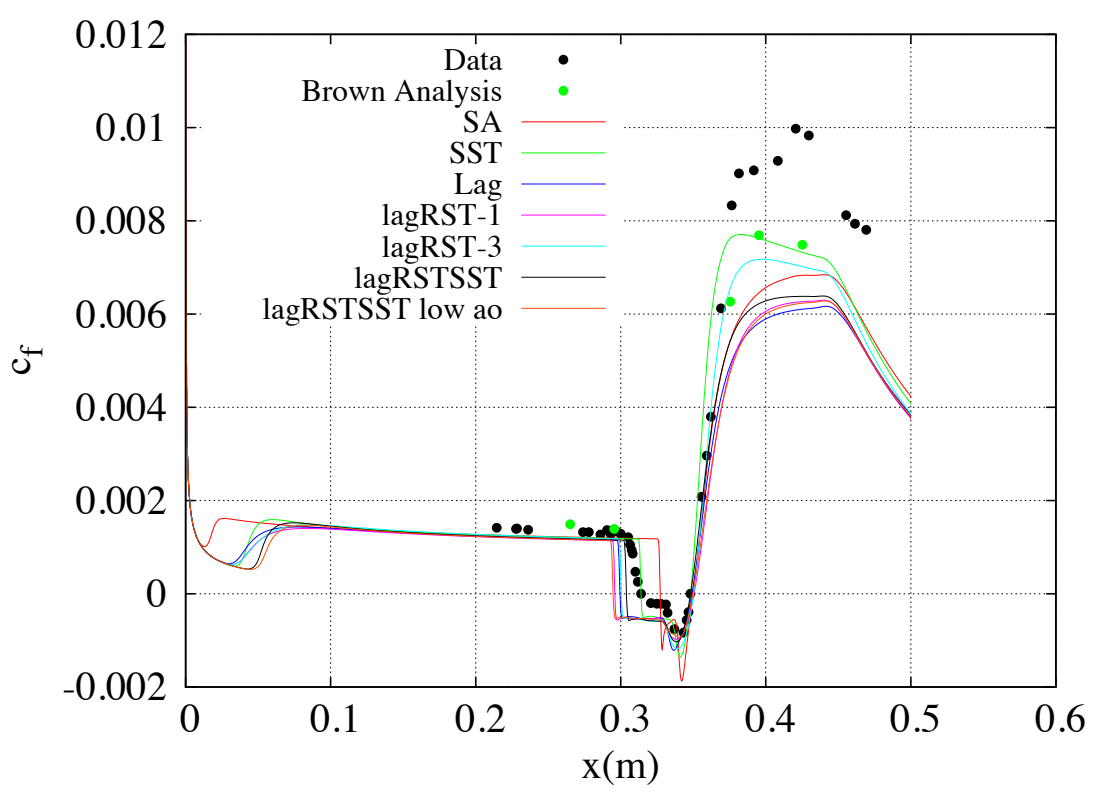

Figure 19. Skin friction distribution comparisons for all turbulence models on the fine grid.

Figure 19 shows the skin friction distributions for all models run. Just as the comparisons to the undisturbed boundary layer showed (figure ??), the skin friction is well under-predicted by all models. It is worth noting that Brown ${ }^{34}$ presents the same under-prediction for this case. Brown used a second method to calculate the skin friction, which brought the skin friction results down by $20 \%-30 \%$. He analyzed the log-law region of pitot probe surveys, which he claims provides an equally accurate result in areas where the flow is attached. For this particular case, he believes that there may be instabilities in the oil flow due to the high shear rates in this area, which would lead credence to the delta between the two methods. The green dots show these results, which do have excellent agreement with the SST model. In this plateau region, the SST model has the largest deviation from the other models. The lag-based models all have the same shape, with the lagRSTSST having a slightly higher skin friction than the $k-\omega$ based models, or the lagRSTSST with the lag constant, $a_{o}$, set to 0.2 .

Figure 20 shows the heat flux comparisons for all the models. All of the models over predict the heat flux by at least $25 \%$, if not substantially more. The SST model has the worst behavior just downstream of the reflected shock, showing an extremely large overshoot in this region. The lag-based models have this overshoot, but the magnitude is much smaller. The SA model does not have this overshoot, but still overpredicts the heat flux. One important item to notice from figure 20 is that the lagRSTSST model largely removes the overshoot of the SST model. In fact, towards the end of the plateau region, the two models predict the same heat flux. So although lagging the Reynolds stress negatively affects the separation zone prediction, it makes a large improvement on the over prediction of heat flux. Note the heat flux measurements have an observed uncertainty of $\sim 20 \%$. 


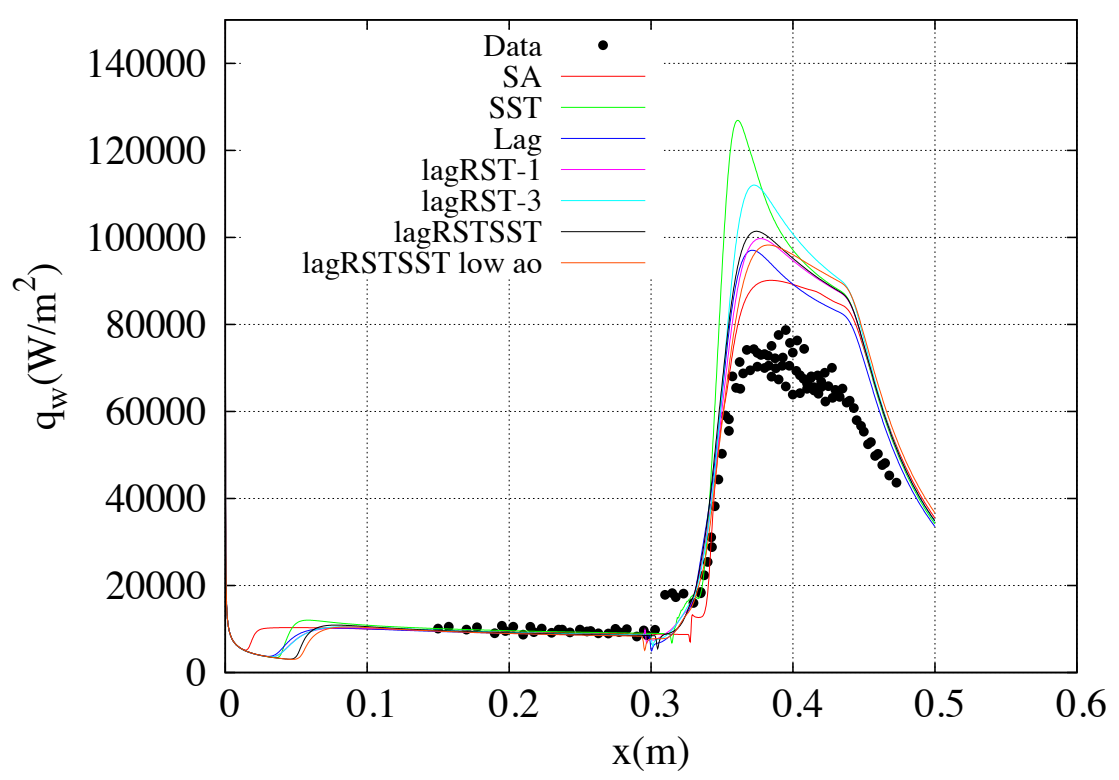

Figure 20. Heat flux $\left(\mathrm{W} / \mathrm{m}^{2}\right)$ distribution comparisons for all turbulence models on the fine grid.

\section{II.F. Orion Capsule}

As part of the CEV (now Orion) program, an experimental study 40,41 of the CEV geometry (shown in figure 21) was conducted at the Ames Unitary Plan Wind Tunnel (UPWT) ${ }^{42}$ in the 11-foot Transonic Test Section and the 9X7 foot Supersonic Test Section. Test data were obtained for conditions at $0.3 \leq M_{\infty} \leq 2.5$, $140^{\circ} \leq \alpha \leq 170^{\circ}$, and $1.0 \times 10^{6} \leq R e_{D} \leq 5.3 \times 10^{6}$. The angle of attack was focused around the trim condition. Three models were constructed during this test, one $7.66 \%$ scale and two $3.0 \%$ scale models. The larger model included pressure taps, unsteady pressure transducers, and an internal balance. One $3.0 \%$ model was designed exclusively for force and moment data (internal balance), and the other was designed solely for surface pressure data. The 3\% models provided Reynolds number comparison, and allowed comparison between test run in two different facilities, which helped quantify uncertainty. The $7.66 \%$ scale model had both pressure taps and an internal balance. Boundary layer trips were applied to the model using CadCut trip dot tape. This study simulated the $R e_{D}=5.3 \times 10^{6}$ case at $M \infty=0.95$. This condition was investigated only with the larger model in the 11-foot Transonic Test Section. Three different angles of attack were assessed, $142.4^{\circ}, 154.4^{\circ}$, and $170.4^{\circ}$. Note that $\alpha=180^{\circ}$ would have the heatshield into the wind and $\alpha=0^{\circ}$ would be the cone into the wind. Through unpublished results and internal reports, the Orion Aerosciences team has shown that the choice of turbulence model makes a large difference on the wake behavior, which directly affects the $C_{D}$ of the capsule. Therefore, this study will focus on comparisons with $C_{D}$ and $C_{L}$. In addition, pitch plane contour images are used to show the wake size and characteristics.

\section{II.F.1. Grid System}

The grid system used was the same grid system employed by Olsen et al. ${ }^{29}$ The capsule is modeled by a three zone grid system, shown in figure 22. The near body grid system is made up of an axi-symmetric grid (in $\mathrm{y}-\mathrm{z}$ ) that is rotated around the $\mathrm{x}$-axis and two H-grids that are overset on the axis points to remove the singularities from the grid-system. The standard overset grid methodology within OVERFLOW ${ }^{43}$ was used to generate the near body grids. The maximum $y^{+}$of the grid systems were $1.5,0.7$, and 0.35 for the coarse, medium, and fine grids, respectively. The near field grid system extended out approximately $\frac{1}{2} \mathrm{D}$ from the capsule. The near body grids were then enclosed with cartesian box grids using the off-body grid generation capability internal to OVERFLOW 2.2c. ${ }^{44,45}$ The wake box grid that enclosed the near body grids extended upstream of the capsule a little less than two capsule diameters and downstream over five capsule diameters. 


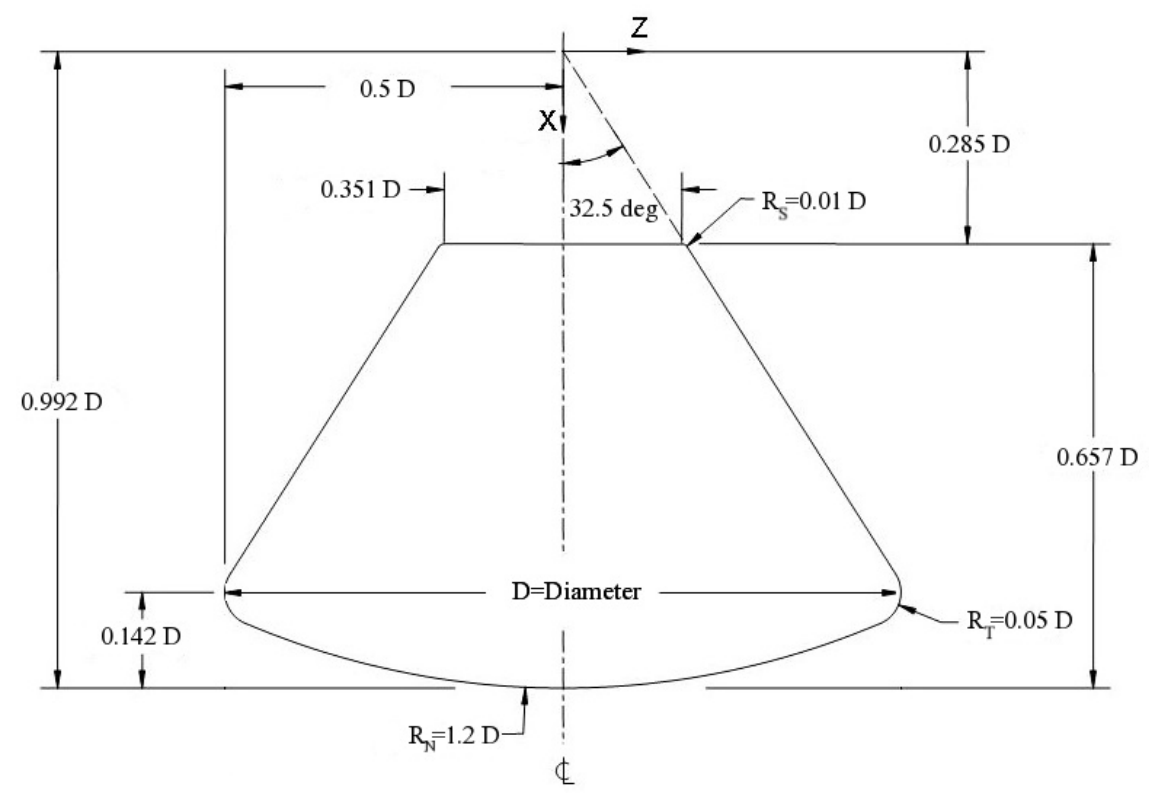

Figure 21. Outer mold line geometry

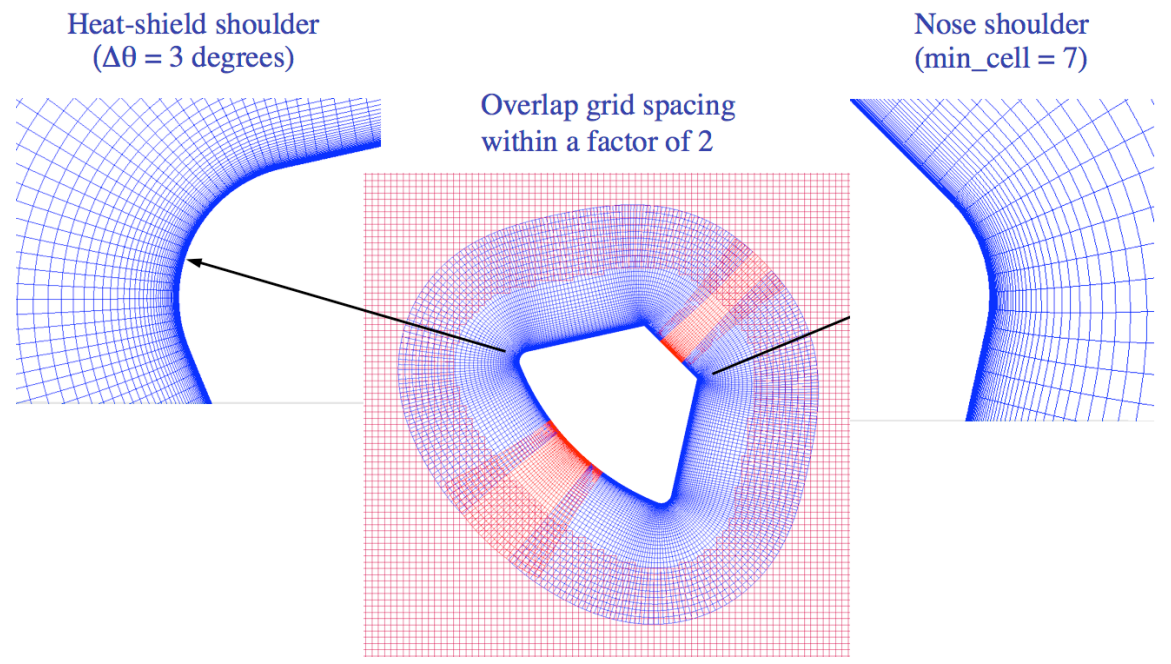

Figure 22. Near body grid system 
This wake box grid was constructed of equispaced cells in all directions. The coarse, medium, and fine grids has $\Delta$ values of 10,5 , and 2.5 inches, respectively. The automatic techniques generated 30,36 , and 45 box grids for the coarse, medium and fine grids, respectively. An image of the medium grid system is shown in figure 23. The domain extended approximately 16 capsule diameters in each direction. The total grid dimensions are listed in table 10.

Table 10. Number of grid points for the Orion grid system.

\begin{tabular}{|c|c|c|c|}
\hline Grid System & Capsule Grids & Box Grids & Total \\
\hline coarse & 230,352 & $1,014,386$ & $1,244,738$ \\
medium & $1,788,565$ & $4,636,564$ & $6,425,129$ \\
fine & $13,402,440$ & $32,323,258$ & $45,725,698$ \\
\hline
\end{tabular}

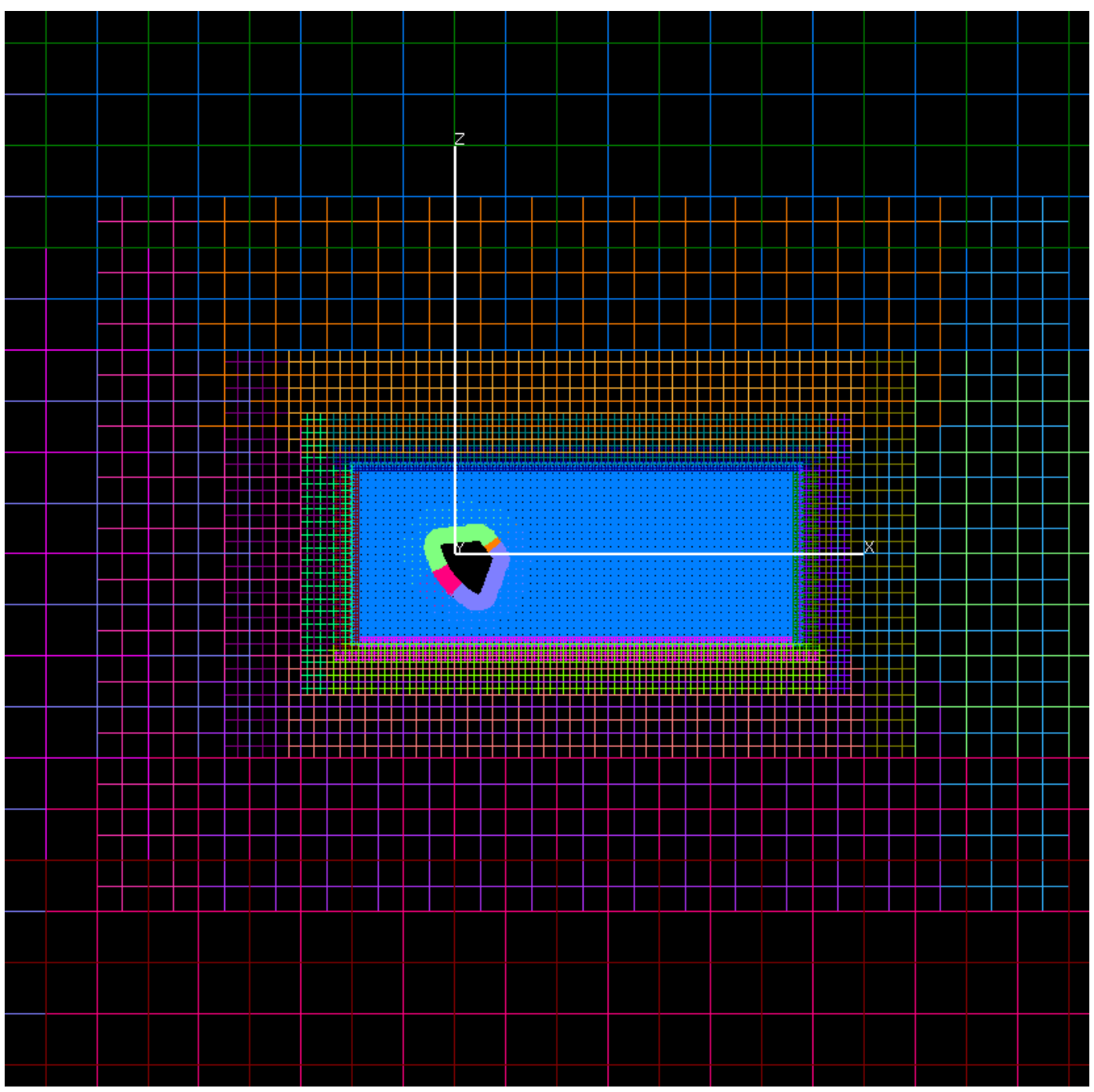

Figure 23. Cartesian box grids enclosing the Orion near body grid system.

\section{II.G. Solution Procedure}

Simulations were initialized using multigrid and grid sequencing and completed with a time accurate simulation using dual time integration to assess the unsteadiness of the flowfields. The time histories of the integrated forces were used to determine whether the flowfields were steady or unsteady. The integrated 
forces also guided judgement about convergence and solution quality. Previous work by Olsen et al. ${ }^{29}$ showed that in general, supersonic cases were steady, and subsonic cases unsteady. This statement came from comparisons for a set of supersonic cases $\left(M_{\infty}=1.4\right)$ and a set of subsonic cases $\left(M_{\infty}=0.95\right)$. Since this study will concentrate on the $M_{\infty}=0.95$ cases, all cases will be simulated assuming unsteady flow conditions.

\section{II.G.1. Grid Convergence}

Due to the significant amount of computer time taken to run the cases, grid convergence was not assessed for every turbulence model. Grid convergence was assessed for the lagRST-1 model at all three chosen angles of attack, while the lagRSTSST model was only assessed at $154.4^{\circ}$ and $170.4^{\circ}$.

Table 11. $C_{L}$ and $C_{D}$ for the $\alpha=170.4^{\circ}$ set of cases.

\begin{tabular}{|c|c|c|c|}
\hline Model & grid & $C_{D}$ & $C_{L}$ \\
\hline lagRST-1 & coarse & 1.10 & 0.18 \\
lagRST-1 & medium & 1.08 & 0.17 \\
lagRST-1 & fine & 1.09 & 0.18 \\
\hline lagRSTSST & coarse & 1.22 & 0.20 \\
lagRSTSST & medium & 1.19 & 0.19 \\
lagRSTSST & fine & 1.19 & 0.20 \\
\hline
\end{tabular}

Table 11 shows the $C_{L}$ and $C_{D}$ for $\alpha=170.4^{\circ}$. The results show for both the lagRST-1 and the lagRSTSST models that grid convergence is obtained with the medium grid. Even the coarse grid results are nearly identical to the medium grid. It is expected that this is due to both models producing steady solutions at this condition. The experimental results provided unsteady wake results, but none of the computations exhibited this behavior. This will be discussed in upcoming sections. The extent of the wake grid was also assessed by increasing its downstream distance by $20 \%$. This had less than a $1 \%$ change on $C_{D}$ or $C_{L}$ and did not affect the unsteadiness of the wake.

Table 12. $C_{L}$ and $C_{D}$ for the $\alpha=154.4^{\circ}$ set of cases.

\begin{tabular}{|c|c|c|c|}
\hline Model & grid & $C_{D}$ & $C_{L}$ \\
\hline lagRST-1 & coarse & 1.09 & 0.49 \\
lagRST-1 & medium & 1.06 & 0.46 \\
lagRST-1 & fine & 1.04 & 0.46 \\
\hline lagRSTSST & coarse & 1.06 & 0.49 \\
lagRSTSST & medium & 1.03 & 0.46 \\
lagRSTSST & fine & 1.01 & 0.45 \\
\hline
\end{tabular}

Table 12 shows the $C_{L}$ and $C_{D}$ for $\alpha=154.4^{\circ}$. Again, the medium and fine grid results are nearly identical, but for this case, the coarse grid results have more variation. The lagRST-1 model did produce an unsteady result, whereas the lagRSTSST model produced a steady result. The medium grid provides a grid converged result.

Table 13. $C_{L}$ and $C_{D}$ for the $\alpha=142.4^{\circ}$ set of cases.

\begin{tabular}{|c|c|c|c|}
\hline Model & grid & $C_{D}$ & $C_{L}$ \\
\hline lagRST-1 & coarse & 1.02 & 0.65 \\
lagRST-1 & medium & 0.87 & 0.59 \\
lagRST-1 & fine & 0.88 & 0.60 \\
\hline
\end{tabular}

Table 13 shows the $C_{L}$ and $C_{D}$ for $\alpha=142.4^{\circ}$. Due to computing constraints, the lagRST- 1 model was the only one used for this $\alpha$. The coarse grid results for this case are very different than the medium and fine. 
The medium and fine grid provide very similar results. This will be discussed more in upcoming sections, but the large deviation from the coarse grid is most likely due to the solution becoming more unsteady as the $\alpha$ gets lower. The numerical oscillations were larger at this condition, and the experimental results showed more unsteadiness. Based on this set of comparisons, the medium grid provides a grid converged solution and will be used for comparisons to the experimental data.

\section{II.G.2. Flowfield Characteristics}

Results were obtained on the medium grid at the three previously discussed $\alpha$ conditions for a suite of turbulence models, ranging from baseline models to the lag-based models. The standard OVERFLOW versions of SA and SST were run along with the standard Lag model. In addition, the lagRST-1, lagRST2 , and lagRST-3 models were run along with the lagRSTSST model with the normal lag constant value $\left(a_{o}=0.35\right)$ and a lower value of the lag constant, $a_{o}=0.2$. All results were averaged over a time interval containing a minimum of two wake oscillations. If an oscillation was not identified (either because the result was steady or the oscillation was not periodic), the time averaging interval was 3000 iterations. With the chosen time advancement parameters, a typical oscillation of the wake took at most 350 iterations.

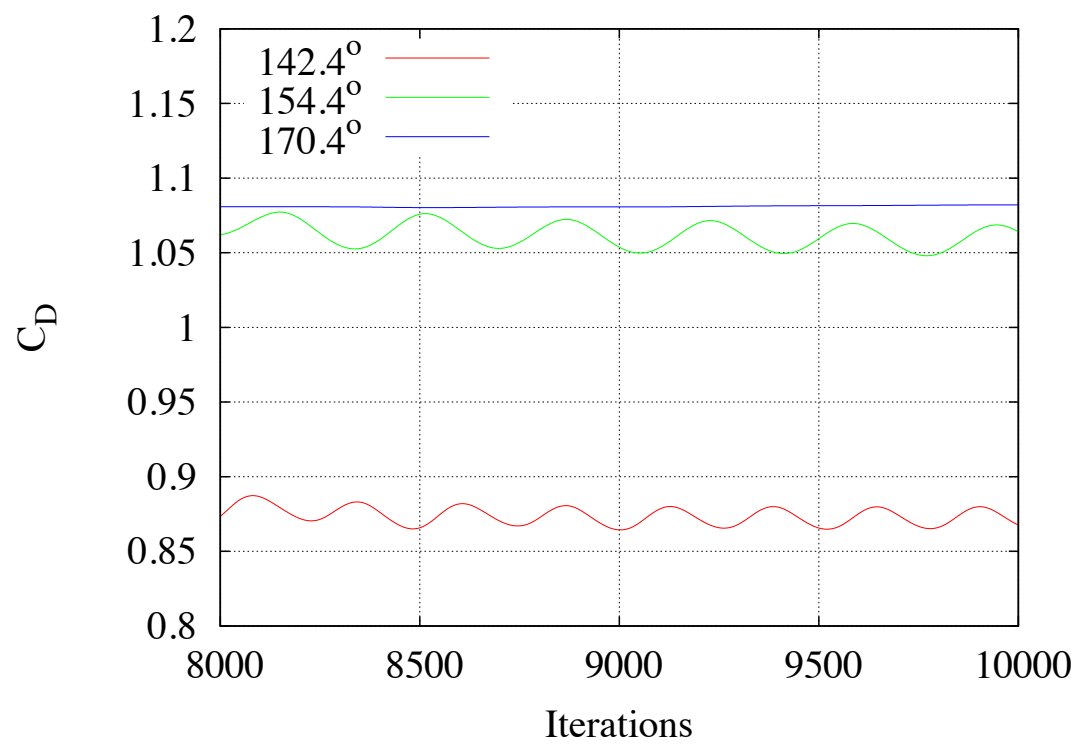

Figure 24. Time history of $C_{D}$ (plotted vs iteration) for the lagRST-1 model at all $\alpha$ 's.

Figure 24 shows a typical time history of $C_{D}$ for the lagRST-1 model at each $\alpha$ (the lagRST-3 model had similar behavior). The oscillation is periodic, indicating a consistent shedding behavior in the wake. The frequency of the oscillation increases going from $\alpha=142.4^{\circ}$ to $\alpha=154.4^{\circ}$. The amplitude of the oscillation goes to zero as the $\alpha$ approaches $170.4^{\circ}$. Once at the highest $\alpha$, the oscillation damps out and the $C_{D}$ results become steady. Only a subset of the models had an oscillating $C_{D}$ at the lower $\alpha$ 's, but every model produced a non-oscillating $C_{D}$ profile at $\alpha=170.4^{\circ}$. Figure 25 shows Mach contours for all three $\alpha$ conditions for the lagRST-1 model. In general, an oscillating $C_{D}$ profile correlates to a wake that is shedding. This can be seen by comparing figures $25(\mathrm{a})$ and $25(\mathrm{~b})$ with figure $25(\mathrm{c})$. At $\alpha=170.4^{\circ}$, the $C_{D}$ time history is steady, and the wake is symmetric and has no shedding. At the two lower $\alpha$ 's, the Mach contours show an asymmetric wake that is shedding, which correlates to previously seen oscillating $C_{D}$ time history. This relationship will generally be the case for all solutions. If the wake is shedding, the $C_{D}$ time history will be oscillating. There is however, one exception to this. Figure 26 compares the Lag model with the lagRST-1 model at $\alpha=142.4^{\circ}$. The Lag model has a nearly steady $C_{D}$ time history (variation in $C_{D}$ of less than $0.5 \%$ ), but figure 26 (a) clearly shows the wake is oscillating. If you compare figure 26(a) with figure 26(b), it is apparent that the re-circulation zone in the wake of the capsule is closing for the Lag model 
before the shedding is occurring. In contrast, the lagRST-1 simulation does not predict the re-circulation zone to close before the shedding begins. It stands to reason that if the re-circulation zone is closed, then the $C_{D}$ time history will be nearly steady even if the wake oscillates downstream of the zone closure.

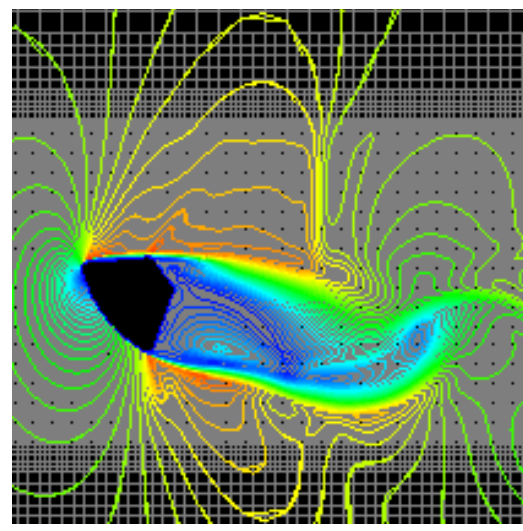

(a) $142.4^{\circ}$

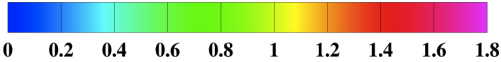

(d) Mach Contour Legend

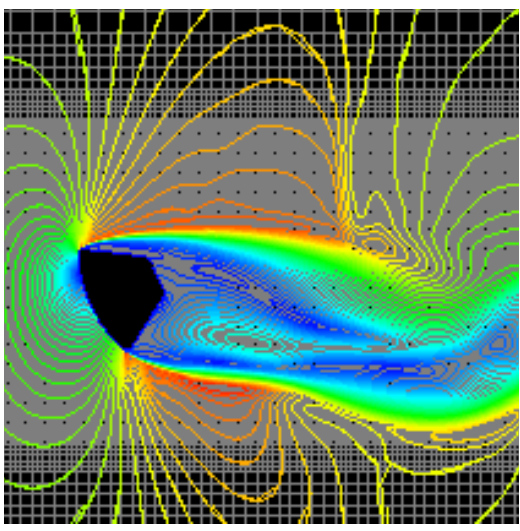

(b) $154.4^{\circ}$

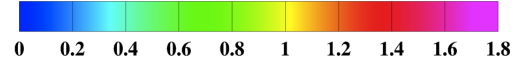

(e) Mach Contour Legend

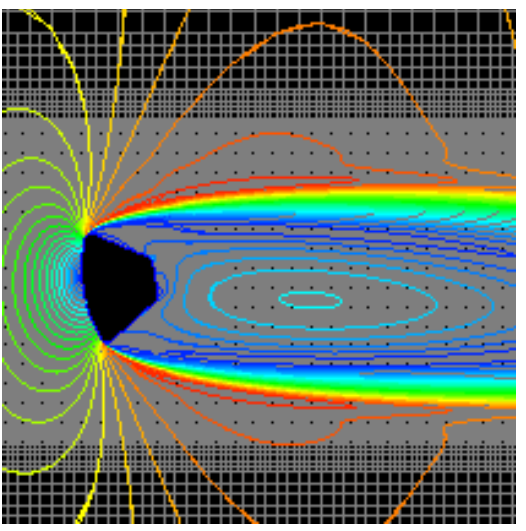

(c) $170.4^{\circ}$

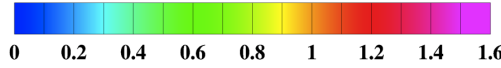

(f) Mach Contour Legend

Figure 25. Mach contours in the pitch plane for the lagRST-1 model showing the wake at all three $\alpha$ 's.

In contract to the lagRST-1 model, the lagRST-2 model shows an unsteady but non-periodic behavior in $C_{D}$. Figure 27 shows the $C_{D}$ time history for over 2000 iterations, which relates to approximately 5 - 10 oscillations of the wake depending on the condition. The solutions are not periodic and are not oscillating about a mean value. Although the results will not be shown here, a more detailed study of this model and its convergence behavior was done. It was found that the model does not produce the expected periodic behavior and the model has difficulty converging to a mean value. This behavior is most likely caused by the lower value of $a_{o}$ used in the lagRST-2 model $\left(a_{o}=0.2\right)$. For the lagRST implementation, this choice of coefficient causes convergence issues and is not recommended for future use.

\section{II.G.3. Comparison with Experiment}

Table 14 shows the $C_{L}$ and $C_{D}$ results for all turbulence models run on the medium grid and the percent difference from the wind tunnel results. The SA and SST models by far have the highest percent error at the lower $\alpha$ 's. Figure 28 compares Mach contours for the the Spallart Allmaras and SST results at $\alpha=142.4^{\circ}$ to the Lag model, which has the lowest percent error. The SA model has a noticeably thicker wake. This larger wake increases the disturbance to the flowfield, and thus increases the $C_{D}$ (indicated by a very large negative percent error). The Spallart Allmaras and SST models also both have stronger shocks than the Lag model. This also accounts for higher drag by reducing $P_{t}$. For the SA and SST models, the precent error reduces as the $\alpha$ increases. However, for the SA model, even at $\alpha=170.4^{\circ}$ the predictions are still off by approximately $20 \%$. The baseline SST model provides better results than the Spalart model at all $\alpha$ 's, but still has a percent error between $12-16 \%$ for the two low $\alpha$ cases. The prediction for $\alpha=170.4^{\circ}$ is excellent, with percent error around $5 \%$.

When all three $\alpha$ 's are considered, the Lag model provides the best results. The $C_{D}$ results are nearly identical at the lower $\alpha$ 's, and and the $C_{L}$ results have some of the lowest percent errors at the same conditions. At $\alpha=170.4^{\circ}$, the percent errors raise significantly over the lower $\alpha$ numbers, but still provide a prediction less than $10 \%$.

For the lagRST-1 and lagRST-3 models, the percent errors are of the same order for all $\alpha$ 's. This is a definite improvement over the SST model for the lower $\alpha$ cases, but for the $\alpha=170.4^{\circ}$ case the errors are larger than the SST model. It is also worth noting that the simulations predict a $C_{D}$ and $C_{L}$ above the experimental results for the lower $\alpha$ cases, but predict a value below the experimental results for the highest $\alpha$. 


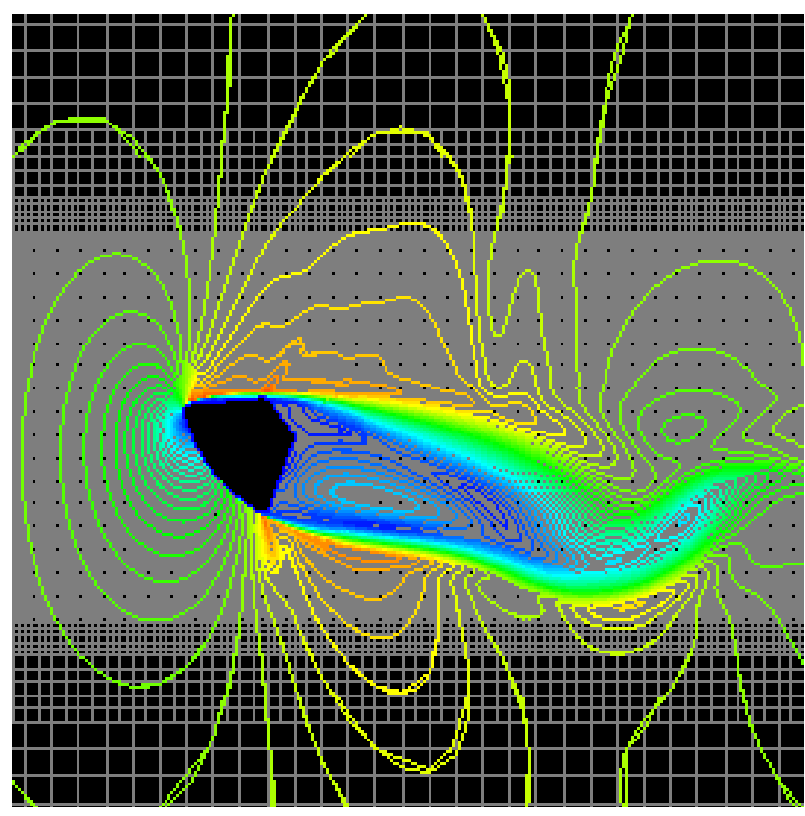

(a) Lag

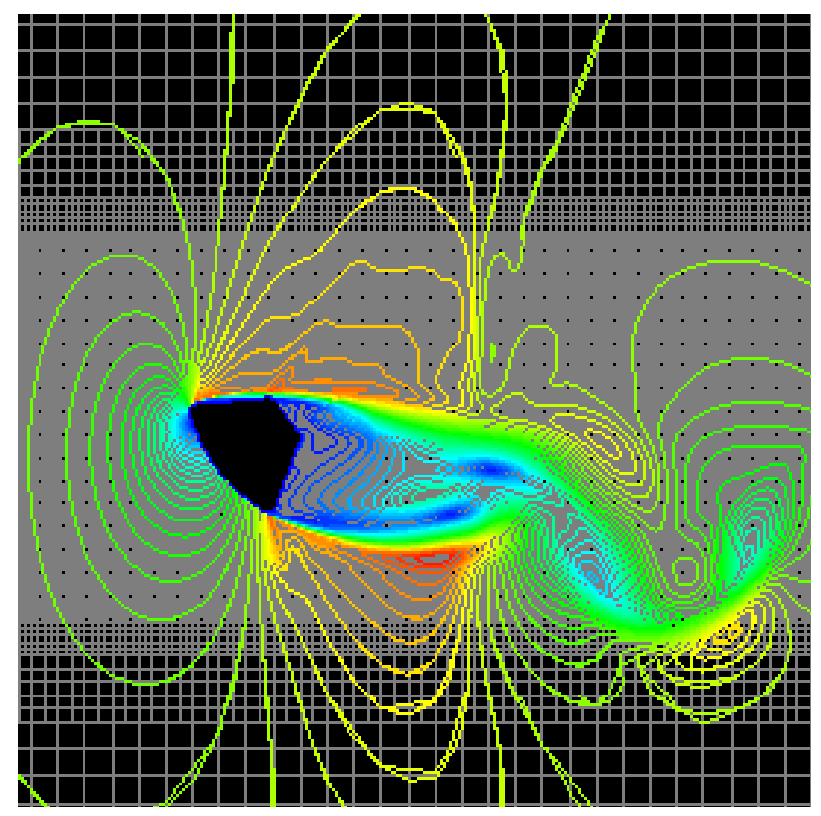

(b) lagRST-1

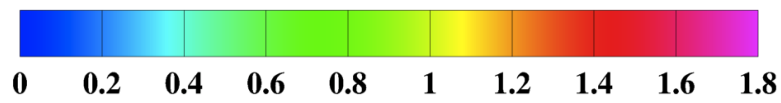

(c) Mach Contour Legend

Figure 26. Mach contours in the pitch plane for the lag and lagRST-1 models showing the wake at $\alpha=142.4^{\circ}$.

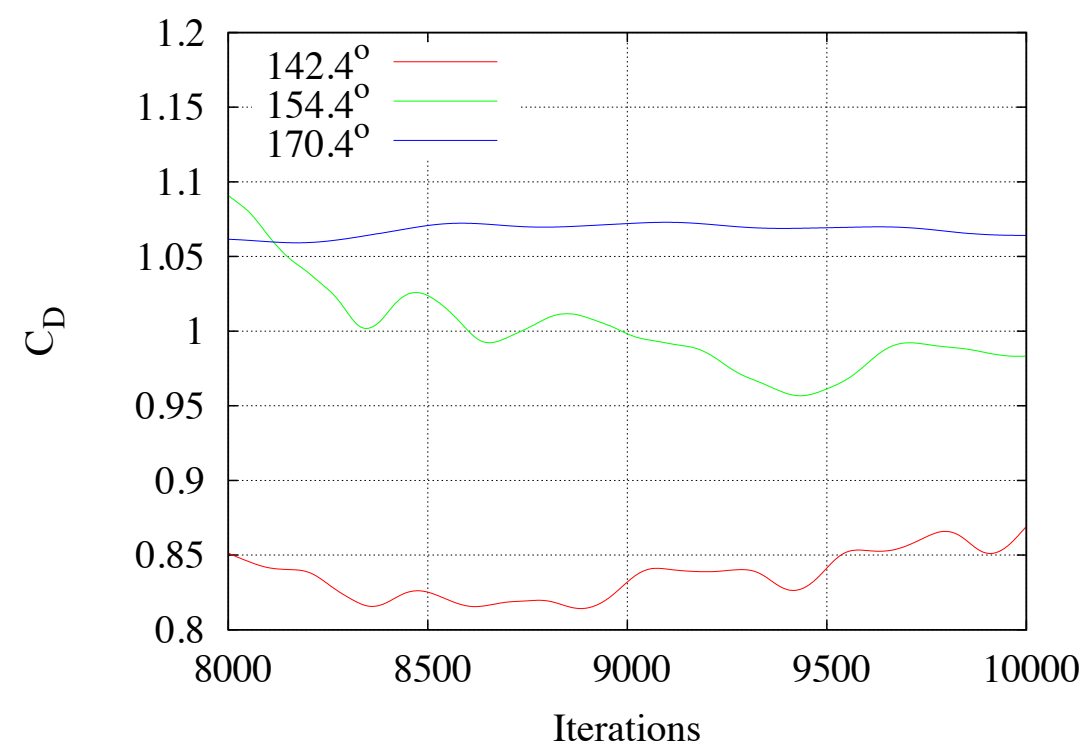

Figure 27. Time history of $C_{D}$ (plotted vs iteration) for the lagRST-2 model at all $\alpha$ 's. 


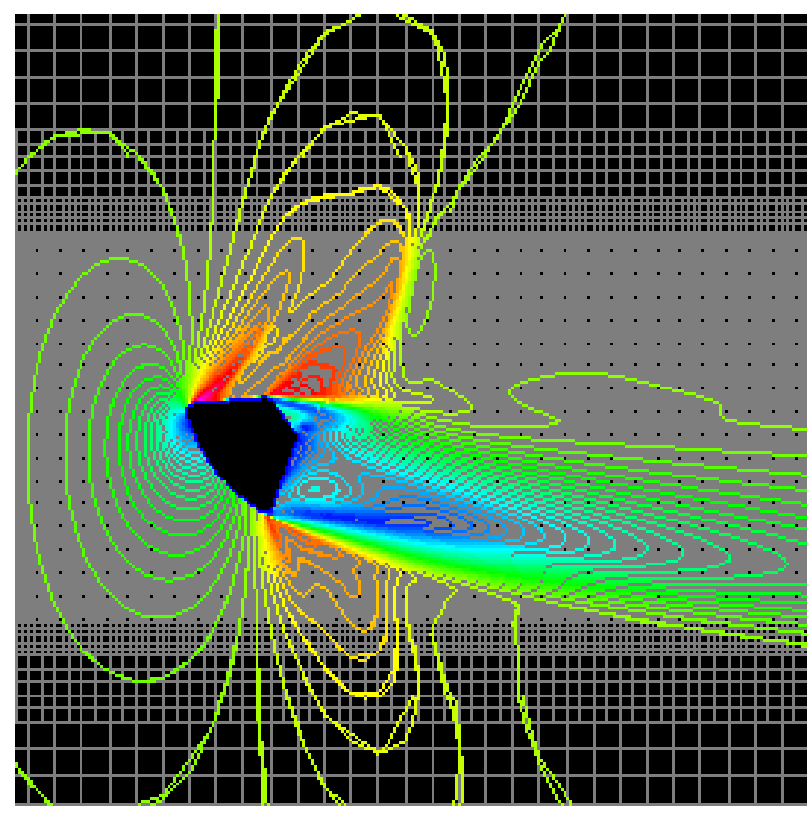

(a) SA

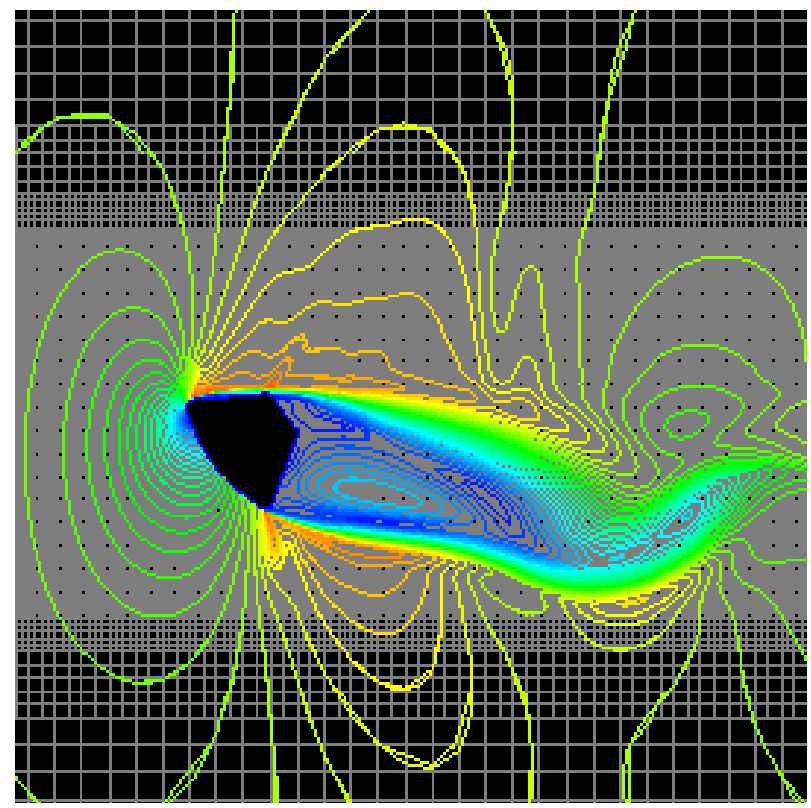

(c) Lag

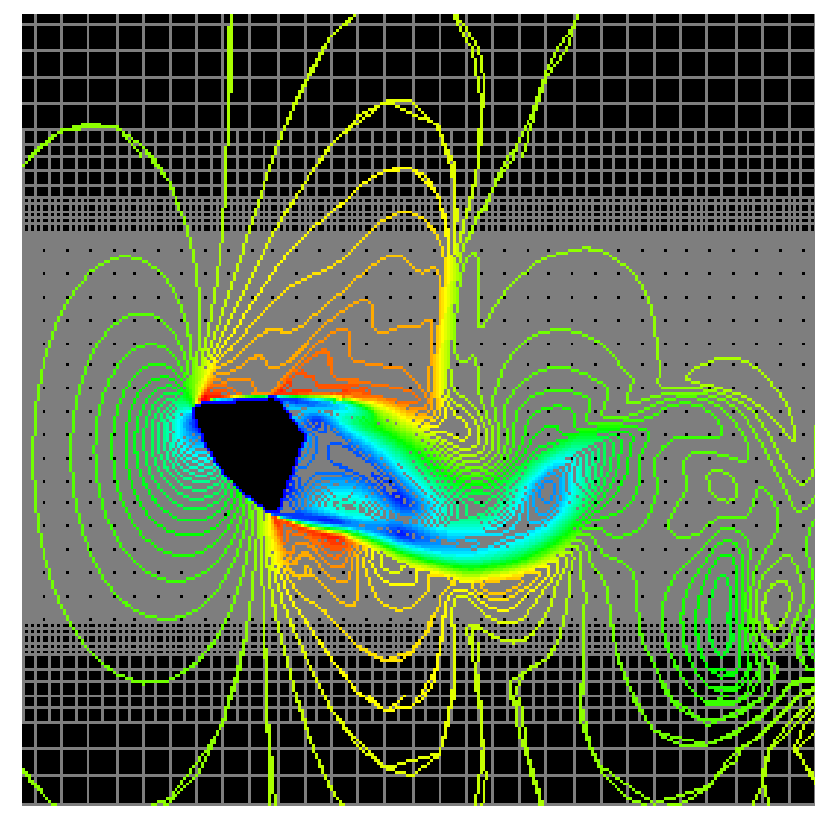

(b) SST

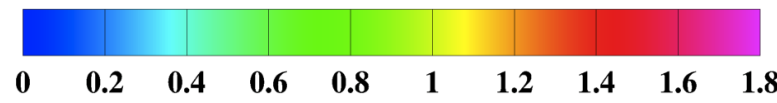

(d) Mach Contour Legend

Figure 28. Mach contours in the pitch plane for the SA and SST models showing the wake at $\alpha=142.4^{\circ}$. 
The lagRSTSST has the best prediction of any model at $\alpha=170.4^{\circ}$, and the percent errors at the two lower $\alpha$ 's are as good or better than any of the other models, excluding the Lag model. Even though $C_{D}$ and $C_{L}$ results are very close for each $\alpha$, the predictions have a non-oscillating wake. The lagRSTSST model is a huge improvement over the baseline SST, especially at the lower $\alpha$ 's, because the percent error for $C_{D}$ reduces by almost $10 \%$ at $\alpha=170.4^{\circ}$. Although the SST model has a small percent error to begin with, the lagRSTSST reduces the \% error by around a factor or two over the baseline SST model. Figure 29 shows four different predictions with SST based models. As previously discussed, the baseline SST provided an unsteady wake, whereas the lagRSTSST does not (this is compared in figure 29(a) with figure 29(c)). At first glance, this behavior is inconsistent with the assumed behavior of the lag-based models. However, there is a valid explanation. The baseline SST model used in these results is the implemented version in OVERFLOW 2.2c, however the SST model used to define the equilibrium conditions for the lagRSTSST model is taken from OVERFLOW 2.0aa. Figure 29(b) shows an SST result with the OVERFLOW 2.0aa implementation, but with the new version of the code. This explains the previous inconsistency. The newer implementation of the SST model produces an unsteady answer. The differences are in the form of limiters in the $2.2 \mathrm{c}$ version of the code that affect the value of $\omega$ in the source calculations, production of $\mathrm{k}$, and the cross diffusion.

Because the lagRSTSST results were steady, a second set of cases using a lower value of $a_{o}$ value $\left(a_{o}=0.2\right)$ were run. With the lower value of $a_{o}$, it was postulated that the results could become unsteady and thus more representative of the wind tunnel test. The results for this test case are labeled "lagRSTSST low $a_{o}$ " in table 14. The $C_{L}$ and $C_{D}$ results are nearly identical to the lagRSTSST model. However the force and moment data exhibit a periodic oscillation. Figure 29 shows the Mach contours comparing the lagRSTSST model with the nominal and lower value of $a_{o}$. Although the $C_{D}$ and $C_{L}$ results are very similar, the wake contours are not. Figure 29(d) shows that by reducing the value of $a_{o}$, the wake near the point where re-circulation would close becomes unsteady and sheds. This is further downstream than what the baseline SST model shows and is slightly upstream of what the Lag model predicts.

Table 15 shows the computed Strouhal numbers for the cases that had oscillating wakes. The number in parenthesis is the percent of the amplitude of the oscillation referenced to the $C_{D}$. The Strouhal number for the numerical simulations was extracted by analyzing the $C_{D}$ and $C_{L}$ time varying results. The difference in physical time between peak to peak oscillations in $C_{D}$ was used to determine the frequency of oscillation. The results was then verified with $C_{L}$, which produced nearly identical results. For the wind tunnel data, time varying $C_{D}$ or $C_{L}$ results were not available, so the Strouhal number was extracted from unsteady pressure data taken from pressure transducers on the backshell. None of the predictions had oscillations at $170.4^{\circ}$. The lagRST-1, lagRST-3, Lag, and SST models all had a repeatable oscillation pattern in the wake for $\alpha$ 's of $142.4^{\circ}$ and $154.4^{\circ}$. The lagRSTSST model did not have an oscillating wake when using an $a_{o}$ value of 0.35 , but when it was reduced to 0.2 , the solution oscillated around a mean value. However, the magnitude of the oscillation was approximately $0.01 \%$ of the total $C_{D}$. There was no oscillation at $\alpha=154.4^{\circ}$ for this model. In general, the results provided by the models are comparable between models. At the lowest $\alpha$, the lagRST-1, lagRST-3, and SST models all have about the same prediction. This is not true for the lagRSTSST (using low $a_{o}$ ), which predicts a lower value that is more comparable to the experimental results. However at the two higher $\alpha$ 's, there is no oscillation.

\section{Summary}

The goal of the current research was to advance current turbulence modeling capabilities in the prediction of shock wave turbulent boundary layer interactions and flows with massive separation for complex configurations, relevant to NASA. The current methodology involved taking a baseline turbulence model $(k-\omega$ or SST) and using it to define an equilibrium turbulent condition. The Reynolds stresses were then relaxed towards this equilibrium condition with a simple "lag" equation, thereby mimicking the expected behavior through which the streamline history influences the turbulence strain rate. This new turbulence model addresses deficiencies observed in flows where varying adverse pressure gradients are seen. The novel turbulence model extends work by Olsen and Coakley ${ }^{7}$ and Olsen et al. ${ }^{19,29}$ to include Reynolds stress transport. By solving for the Reynolds Stresses and not the turbulent eddy viscosity, the models are allowed to relax to their non-equilibrium values with more degrees of freedom. This work represents one of the first set of computations with a robust Reynolds stresses model in a production CFD code applied to real geometries of interest in a URANS method. 


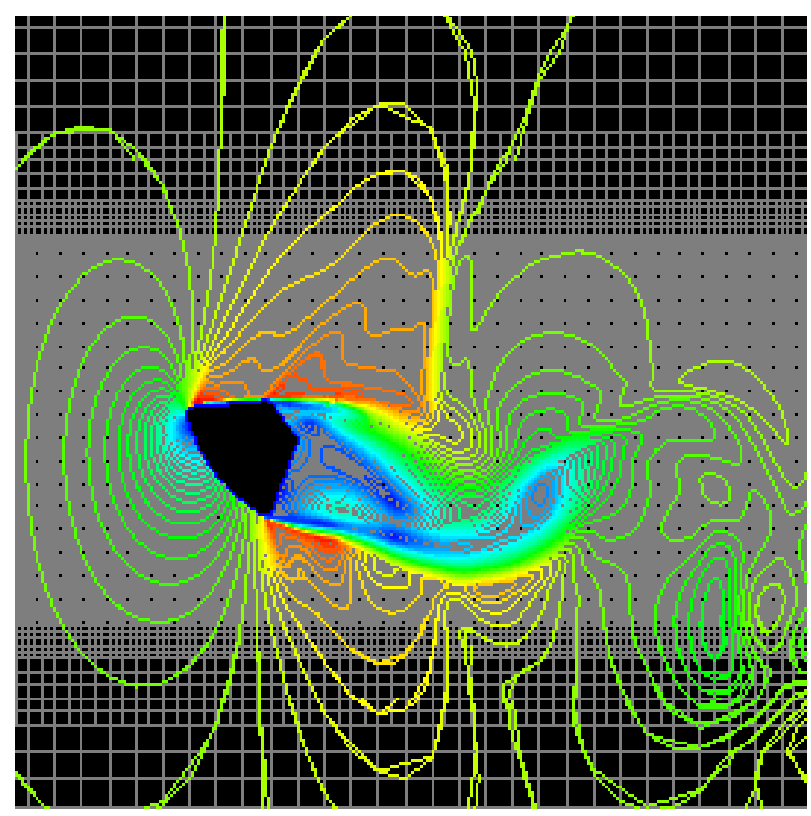

(a) SST OVERFLOW V2.2

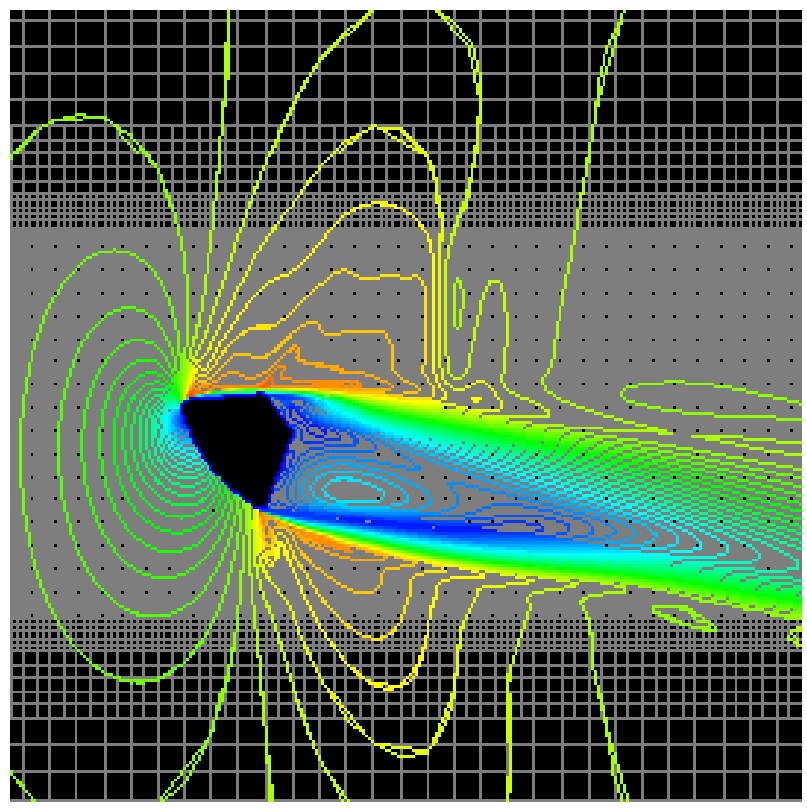

(c) lagRSTSST

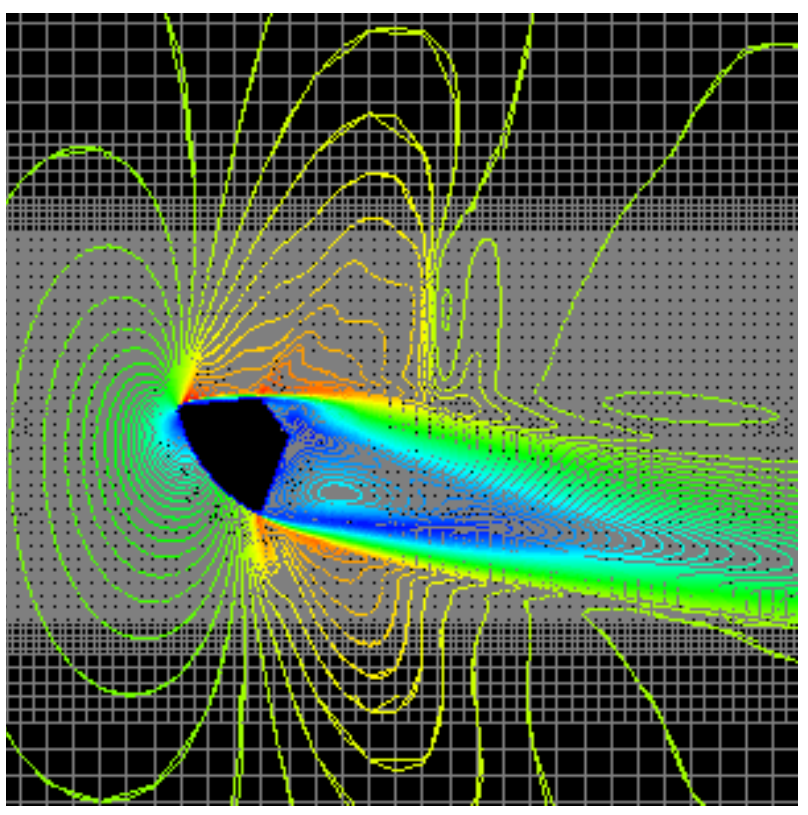

(b) SST OVERFLOW V2.0

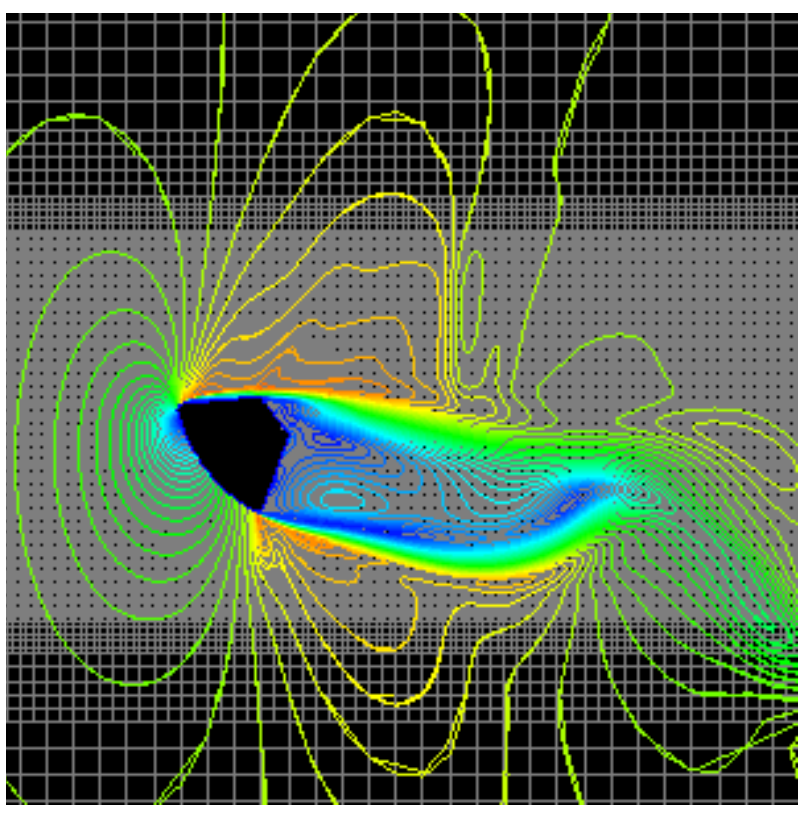

(d) lagRSTSST low $a_{o}$

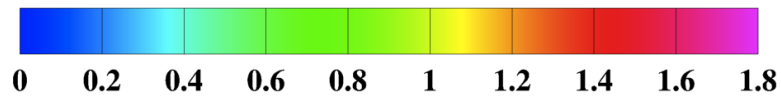

(e) Mach Contour Legend

Figure 29. Mach contours in the pitch plane for the SST and lagRSTSST model with the nominal and lower values of $a_{o}$ showing the wake at $\alpha=142.4^{\circ}$. 
Table 14. $C_{L}$ and $C_{D}$ results and percent errors for all turbulence models on the medium grid.

\begin{tabular}{|c|c|c|c|c|c|}
\hline Model & Alpha & $C_{D}$ & $C_{L}$ & $C_{D} \%$ error & $C_{L} \%$ error \\
\hline SA & $142.4^{\circ}$ & 1.04 & 0.71 & -25.8 & -31.5 \\
SA & $154.4^{\circ}$ & 1.23 & 0.58 & -23.8 & 39.2 \\
SA & $170.4^{\circ}$ & 1.39 & 0.23 & -19.4 & -18.2 \\
\hline SST V2.2 & $142.4^{\circ}$ & 0.95 & 0.63 & -15.2 & -16.2 \\
SST V2.2 & $154.4^{\circ}$ & 1.12 & 0.49 & -12.6 & -16.1 \\
SST V2.2 & $170.4^{\circ}$ & 1.11 & 0.18 & 4.1 & 5.5 \\
\hline Lag & $142.4^{\circ}$ & 0.83 & 0.58 & -0.9 & -7.1 \\
Lag & $154.4^{\circ}$ & 0.98 & 0.44 & 0.7 & -4.4 \\
Lag & $170.4^{\circ}$ & 1.09 & 0.17 & 6.3 & 10.0 \\
\hline lagRST-1 & $142.4^{\circ}$ & 0.87 & 0.59 & -5.7 & -9.0 \\
lagRST-1 & $154.4^{\circ}$ & 1.06 & 0.46 & -6.7 & -10.2 \\
lagRST-1 & $170.4^{\circ}$ & 1.08 & 0.17 & 6.7 & 12.1 \\
\hline lagRST-3 & $142.4^{\circ}$ & 0.87 & 0.59 & -5.2 & -7.8 \\
lagRST-3 & $154.4^{\circ}$ & 1.06 & 0.46 & -6.9 & -8.7 \\
lagRST-3 & $170.4^{\circ}$ & 1.09 & 0.17 & 6.2 & 12.2 \\
\hline lagRSTSST & $142.4^{\circ}$ & 0.85 & 0.59 & -3.1 & -8.6 \\
lagRSTSST & $154.4^{\circ}$ & 1.03 & 0.46 & -4.4 & -10.1 \\
lagRSTSST & $170.4^{\circ}$ & 1.19 & 0.19 & -2.3 & 0.9 \\
\hline lagRSTSST low $a_{o}$ & $142.4^{\circ}$ & 0.83 & 0.57 & -4.5 & -7.4 \\
lagRSTSST low $a_{o}$ & $154.4^{\circ}$ & 1.01 & 0.44 & -2.3 & -6.0 \\
lagRSTSST low $a_{o}$ & $170.4^{\circ}$ & 1.16 & 0.19 & -0.3 & 4.1 \\
\hline SST V2.0 & $142.4^{\circ}$ & 0.88 & 0.62 & -7.1 & -13.8 \\
SST V2.0 & $154.4^{\circ}$ & 1.07 & 0.49 & -8.3 & -17.3 \\
SST V2.0 & $170.4^{\circ}$ & 1.22 & 0.20 & -5.6 & -4.8 \\
\hline
\end{tabular}

The initial study was on four computationally "simple" test cases, an incompressible flat plate, a turbulent mixing layer, an incompressible adverse pressure gradient turbulent boundary layer with separation, and a transonic bump. The results of this initial study showed mixed results, with no singular implementation of the model providing the best results. All of the implementations worked well for the incompressible turbulent boundary layer, but none of the models were able to predict the proper shape of the velocity profile near the edge. This is not surprising, as typical closure techniques concentrate on matching the wall properties and the law of the wall first. For the mixing layer, the $k-\omega$ based models incorrectly predicted the spreading rate by nearly a factor of two below the experimentally determined value. In contrast, the SST based models predicted a spreading rate that matched the experimental data very well. This result was unexpected and is still being assessed. In contrast, the separation predictions were far more accurate using the $k-\omega$ based models, with the standard lagRST model providing excellent results. The SST based models over predicted the separation zone for both the incompressible and transonic test cases by over $40 \%$.

The major set of analysis done in this project centered around the predictions on two test cases. The first

Table 15. Computed Strouhal number for the lagRST-1, lagRST-3, SST, and lagRSTSST (with $a_{o}=0.2$ ) models. The numbers in parenthesis after the Strouhal number is a measure of the oscillation. It is the percent the amplitude of the oscillation is of the total $C_{D}$.

\begin{tabular}{|c|c|c|c|c|c|c|}
\hline$\alpha$ & Data & lagRST-1 & lagRST-3 & SST & lagRSTSST & Lag \\
\hline $142.4^{\circ}$ & 0.22 & $0.27(1.7 \%)$ & $0.25(1.4 \%)$ & $0.26(2.5 \%)$ & $0.22(0.01 \%)$ & $0.27(0.64 \%)$ \\
$154.4^{\circ}$ & 0.17 & $0.18(1.8 \%)$ & $0.18(1.9 \%)$ & $0.19(2.1 \%)$ & $\mathrm{n} / \mathrm{a}$ & $0.24(0.24 \%)$ \\
$170.4^{\circ}$ & 0.15 & $\mathrm{n} / \mathrm{a}$ & $\mathrm{n} / \mathrm{a}$ & $\mathrm{n} / \mathrm{a}$ & $\mathrm{n} / \mathrm{a}$ & $\mathrm{n} / \mathrm{a}$ \\
\hline
\end{tabular}


test case was a hypersonic SWTBLI of an impinging oblique shock on a fully developed turbulent boundary layer that caused separation. For this test case, the baseline SST model provided separation zone length predictions that were less than $1 \%$ from the experimentally observed values. The lagRSTSST and lagRST models both predict separation zone lengths on the order of $50 \%$ too large. In addition to separation zone accuracy, of particular interest too was the peak heat flux prediction at reattachment. The SST model over predicted this heat flux by $65 \%$. By lagging the Reynolds stresses, the peak heat flux prediction reduced by $25 \%$ for the lagRSTSST. So although the lagging technique had an adverse affect on the prediction of the separation zone, it did have a positive effect on one of the negative characteristics of the SST model for SWTBLI.

The final analysis was done on the Orion (now MPCV) capsule. The grid system and techniques used here are the ones used by NASA's aerodynamics teams building the aerodynamic databases for these vehicles, and the baseline models are the ones used by NASA. The first contribution of this analysis is that the Reynolds stress modeling implemented in the OVERFLOW code is robust and can be run in an unsteady, time accurate fashion and provide grid converged results. This is a significant contribution since the predictions are solving for Reynolds stresses. The results for this case showed excellent results for the lag technique. The standard turbulence models (Spalart Allmaras and SST) almost always provided higher percent errors than the lag based models. The Lag, lagRST, and lagRSTSST models all were able to generally predict the $C_{D}$ and $C_{L}$ to less than $10 \%$. Several of the lag based models predicted oscillating wakes with Strouhal numbers comparable to the experimentally obtained values at lower $\alpha$ 's. Unfortunately, none of the models were able to predict an unsteady wake at the highest $\alpha$.

\section{Recommendations}

It is clear from the analysis done that a simple lag based Reynolds Stress model does not solve all the problems identified for flowfields with massive separation or SWTBLI interactions. Based the suite of cases, two models are recommended for further study. The lagRST-1 model should be treated as the baseline lagRST model, with the same coefficients used in the standard eddy viscosity Lag model, $a_{o}=0.35$ and $\sigma_{k}=0.8$. This model did very well for incompressible and transonic flow predictions. It should be noted that if the flow of interest has a mixing layer component, the predictions need to be validated with experiments because of its poor predictions of the growth rate of the Bell Mehta mixing layer. Although this mixing layer issue needs to be explained, it did not seem to affect the capsule predictions. The lagRSTSST model with the standard coefficients $\left(a_{o}=0.35\right)$ also performed well for the complicated test cases. The issue with this model is its separation prediction for incompressible and transonic flat plates, where the results were very poor. The separation predictions for this model were actually better for the SWTBLI than for the simpler cases, and the lag equations reduced the peak heat flux at the reflected shock. These are promising results, as an over prediction of the magnitude SST had would most likely result in over design of the TPS system.

The SWTBLI results should be verified with other wind tunnel datasets to ensure consistent behavior. Brown $^{34}$ has identified two other high quality experimental datasets that could be used. One dataset is the Mach 8.9 experiment of Murray ${ }^{46-48}$ which was conducted at the Imperial College Nitrogen gun tunnel. This test series consists of a hollow axisymmetric cylinder with an axisymmetric cowl use as a shock ring generator. Data available for this case are wall pressure and heat flux. The second experiment is the Mach 8.18 experiment of Kussoy and Horstman. ${ }^{49}$ It was conducted on a 2-D flat plate in the no longer operational NASA Ames Research Center Hypersonic facility. The test configuration was the nominal 2-D flat plate with a shock generator inclined, similar to the Schulein configuration assessed in this study. Pressure, skin friction, and heat flux were obtained in this study as well as flow field quantities (no turbulence quantities) were obtained. In addition, these configurations should be assessed for 3-D affects.

In addition to simulating more configurations, there are also several other forms of the turbulence model that should be modeled. In particular, the form of the Reynolds stress tensor used in the lagRST model could provide numerical and / or physical modeling improvements over the current implementation. Two of these forms are detailed below. The anisotropy tensor has been used by several other researchers ${ }^{22,23,50,51}$ and may provide improvements to the predictions.

Instead of using $\tau_{i j}$, the mean normal stress component can be subtracted from the Reynolds stress to leave the deviatoric portion of the tensor. The Boussinesq equation (6) can be rewritten to show 


$$
\begin{aligned}
\tau_{i j_{e q}}-\frac{1}{3} \tau_{k k_{e q}} \delta_{i j} & =2 \rho \nu_{t_{e q}}\left(s_{i j}-\frac{1}{3} s_{k k} \delta_{i j}\right), \\
\tau_{i j_{e q}}^{D} & =2 \nu_{t_{e q}} s_{i j}^{D} \rho,
\end{aligned}
$$

where the superscript $\mathrm{D}$ denotes the deviatoric part of the tensor. The lag equation would then become the following.

$$
\frac{\partial \tau_{i j}^{D}}{\partial t}+\frac{\partial}{\partial x_{k}}\left(\tau_{i j}^{D} \tilde{u}_{k}\right)=a_{o} \omega\left(\tau_{i j_{e q}}^{D}-\tau_{i j}^{D}\right)
$$

In this formulation, the turbulent kinetic energy solved for in equation 3 would be the actual lagged variable used in all flowfield computations.

The Reynolds stress anisotropy tensor is another option for the variable to be lagged. It is defined here.

$$
b_{i j}=\frac{\bar{\rho} \widetilde{u_{i}^{\prime} u_{j}^{\prime}}-\frac{1}{3} \bar{\rho} \widetilde{u_{k}^{\prime} u_{k}^{\prime}} \delta_{i j}}{\bar{\rho} \widetilde{u_{l}^{\prime} u_{l}^{\prime}}}
$$

By substituting equations 2 and the relation $\tau_{k k}=-\bar{\rho} \widetilde{u_{k}^{\prime} u_{k}^{\prime}}=-2 \bar{\rho} k$ into the equation for $b_{i j}$, you arrive at

$$
b_{i j}=-\frac{\tau_{i j}-\frac{1}{3} \tau_{k k} \delta_{i j}}{2 \bar{\rho} k} .
$$

The numerator of the previous equation is the deviatoric Reynolds stress tensor (left hand side of equation 13), thus substitution leaves

$$
b_{i j}=-\frac{\tau_{i j}^{D}}{2 \bar{\rho} k}
$$

Equation 14 can then be used to define the $b_{i j_{e q}}$, the equilibrium anisotropic Reynolds stress tensor,

$$
b_{i j_{e q}}=-\frac{\mu_{t} s_{i j}^{D}}{\bar{\rho} k} .
$$

The lag equation would then become

$$
\frac{\partial \bar{\rho} b_{i j}}{\partial t}+\frac{\partial}{\partial x_{k}}\left(\bar{\rho} b_{i j} \tilde{u}_{k}\right)=a_{o} \omega\left(\bar{\rho} b_{i j_{e q}}-\bar{\rho} b_{i j}\right)
$$

Another possibility is to remove the Boussinesq approximation and drive the Reynolds Stress tensor to a higher closure model. This could be done by solving for an algebraic Reynolds Stress tensor.

Coakley $^{52}$ proposed what he refers to as the KEA (the letters KE indicates that this version of his model tries to follow $k-\epsilon$ model behavior and the A represents the choice of switching function used) model, which was intended to address deficiencies in the SST model. Coakley's primary purpose was to remove the dependence on wall distance. This would improve the timings of the lag based models based on the SST formulations, while still keeping the accurate mixing layer predictions that eluded the $k-\omega$ based models. Coakley shows results that are extremely close to the SST predictions with the KEA model.

\section{References}

\footnotetext{
${ }^{1}$ Spalart, P. R. and Allmaras, S. R., "A One-Equation Turbulence Model for Aerodynamic Flows," AIAA 30th Aerospace Sciences Meeting, Reno, NV, January 1992, AIAA 92-0439.

${ }^{2}$ Jones, W. P. and Launder, B. E., "The Prediction of Laminarization with a Two-Equation Model of Turbulence," International Journal of Heat and Mass Transfer, Vol. 15, 1972, pp. 301-314.

${ }^{3}$ Launder, B. E. and Sharma, B. I., "Application of the Energy Dissipation Model of Turbulence to the Calculation of Flow Near a Spinning Disc," Letters in Heat and Mass Transfer, Vol. 1, No. 2, 1974, pp. 131-138.

${ }^{4}$ Wilcox, D. C., Turbulence Modelling for CFD, DCW Industries, Inc., La Canada, CA, 1998.

${ }^{5}$ Rizzetta, D. P., "Evaluation of Explicit Algebraic Reynolds-Stress Models for Separated Supersonic Flows," AIAA Journal, Vol. 36, 1998, pp. 24-30.
} 
${ }^{6}$ Viti, V., Huang, G., and Bradshaw, P., "Implementation and Validation of recent stress-transport turbulence models: the need for standardized procedures," 43rd AIAA Aerospace Science Meeting and Exhibit, Reno, NV, January 2005, AIAA 2005-112.

${ }^{7}$ Olsen, M. E. and Coakley, T. J., "The Lag model, a turbulence model for non equilibrium flows," 15th AIAA Fluid Dynamics Conference, Anaheim, CA, June 2001, AIAA 2001-2564.

${ }^{8}$ Menter, F. R., "Zonal two equation $k-\omega$ turbulence models for aerodynamic flows," July 1993, AIAA paper 93-2906.

${ }^{9}$ Menter, F. R., "Two-Equation Eddy-Viscosity Turbulence Models for Engineering Applications," AIAA Journal, Vol. 32 , No. 8, August 1994, pp. 1598-1605.

${ }^{10}$ Bell, J. H. and Mehtai, R. D., "Development of a Two-Stream Mixing Layer from Tripped and Untripped Boundary Layers," AIAA Journal, Vol. 28, No. 12, December 1990, pp. 2034-2043.

${ }^{11}$ Driver, D. M., "Reynolds Shear Stress Measurements in a Separated Boundary Layer FLow," AIAA 22nd Fluid Dynamics, Plasma Dynamics, and Lasers Conference, Honolulu, Hawaii, June 1991, AIAA 1991-1787.

${ }^{12}$ Bachalo, W. D. and Johnson, D. A., "Transonic, Turbulent Boundary-Layer Separation Generated on an Axisymmetric Flow Field," AIAA Journal, Vol. 24, No. 3, 1986, pp. 437-443.

${ }^{13}$ Moseley, William C., Moore, Robert H. Jr, and Hughes, Jack E., "Stability Characteristics of the Apollo Command Module," NASA TN 3890.

${ }^{14}$ Wilcox, D. C., Turbulence Modelling for CFD, DCW Industries, Inc., La Canada, CA, 2006.

${ }^{15}$ Marshall, T. A. and Dolling, D. S., "Computation of Turbulent, Separated, Unswept Compression Ramp Interactions," AIAA Journal, Vol. 30, 1992, pp. 2056-2065.

${ }^{16}$ Knight, D. and Degrez, G., "Shock wave boundary layer interactions in high Mach number flows: a critical survey of current CFD prediction capabilities," AGARD Advisory Report 319, Vol. II, December 1998, pp. 1-1 to 1-35.

${ }^{17}$ Clauser, F. H., "The turbulent boundary layer," Advanced Applied Mechanics, Vol. 4, 1956, pp. 1-54.

${ }^{18}$ Lillard, R. P., Turbulence Modeling for Shock Wave/Turbulent Boundary Layer Interactions, PhD Thesis, Purdue University, West Lafayette, Indiana, December 2011.

${ }^{19}$ Olsen, M. E., Coakley, T. J., and Lillard, R. P., "The Lag model applied to high speed flows," 43rd AIAA Aerospace Sciences Meeting and Exhibit, Reno, Nevada, January 2005, AIAA 2005-0101.

${ }^{20}$ Buning, P., "NASA LaRC, Private Communication," .

${ }^{21}$ Jespersen, D. C., Pulliam, T. H., and Buning, P. G., "Recent Enhancements to OVERFLOW," AIAA 35th Aerospace Sciences Meeting, Reno, NV, January 1997, AIAA 97-0644.

${ }^{22}$ Hamlington, P. E. and Dahm, W. J., "Reynolds stress closure for nonequilibrium effects in turbulent flows," Physics of Fluids, Vol. 20, No. 115101, 2009.

${ }^{23}$ Hamlington, P. E. and Dahm, W. J., "Computational Validation of New Reynolds Stress Closure for Nonequilibrium Effects in Turbulent Flows," 47th AIAA Aerospace Sciences Meeting Including The New Horizons Forum and Aerospace Exposition, Orlando, FL, January 2009, AIAA 2009-1323.

${ }^{24}$ Churchfield, M. and Blaisdell, G., "The Lag RST Turbulence Model Applied to a Vortex Flow," AIAA 46th Aerospace Sciences Meeting and Exhibit, Reno, NV, January 2011, AIAA 2011-663.

${ }^{25}$ Lillard, R. P., Turbulence Modeling for Shock Wave/Turbulent Boundary Layer Interactions, Preliminary Report, Purdue University, West Lafayette, IN, November 2005.

${ }^{26}$ Churchfield, M. J., The Lag RST Turbulence Model Applied to Vortical Flows, PhD Thesis, Purdue University, West Lafayette, Indiana, August 2009.

${ }^{27}$ Churchfield, M. and Blaisdell, G., "A Reynolds Stress Relaxation Tubulence Model Applied to a Wingtip Vortex Flow," 49th AIAA Aerospace Sciences meeting including the New Horizons Forum and Aerospace Exhibition, January 2008, AIAA 2008-0769.

${ }^{28}$ Olsen, M. E. and Prabhu, D. K., "Application of OVERFLOW to Hypersonic Perfect Gas Flowfields," 15th AIAA Fluid Dynamics Conference, Anaheim, CA, June 2001, AIAA 2001-2664.

${ }^{29}$ Olsen, M. E., Chaderjain, N., Lillard, R. P., Greathouse, J. S., and Coakley, T. J., "Numerical study of massively separated flows," 45th AIAA Aerospace Sciences Meeting and Exhibit, Reno, Nevada, January 2007, AIAA $2007-1412$.

${ }^{30}$ Osterlund, J. M., Experimental Studies of Zero Pressure-Gradient Turbulent Boundary-Layer FLow, PhD Thesis, Royal Institute of Technology, Se-100 44 Stockholm, Sweden, December 1999.

${ }^{31}$ Osterlund, J. M., Johansson, A. V., Nagib, H. M., and Hites, M. H., "Wall shear stress measurements in high Reynolds number boundary layers from two facilities," 30th AIAA Fluid Dynamics Conference, Norfolk, VA, 1999, AIAA 1999-3814.

${ }^{32}$ Schulein, E., Krogmann, P., and Stanewsky, E., "Documentation of Two-Dimensional Impinging Shock/Turbulent Boundary Layer Interaction Flow," Tech. Rep. Rept. 1B 223-96 A 49, DLR, German Aerospace Center, Gottingen, Germany, October 1996.

${ }^{33}$ Schulein, E., "Skin Friction and Heat Flux Measurements in Shock Boundary Layer Interaction Flows," AIAA Journal, Vol. 44, No. 8, 2006, pp. 1732-1741.

${ }^{34}$ Brown, J. L., "Shock Wave Impingement on Boundary Layers at Hypersonic Speeds: Computational Analysis and Uncertainty," 42nd AIAA Thermophysics Conference, 27 - 30 June 2011, Honolulu, HI, June 2011, AIAA $2011-3143$.

${ }^{35}$ Fedorova, N. N., Fedorchenko, I. A., and Schulein, E., "Experimental and Numerical Study of Oblique Shock Wave/Turbulent Boundary Layer Interaction at M = 5," Computational Fluid Dynamics Journal, Vol. 10, No. 3, 2001, pp. 376183, Special Issue.

${ }^{36}$ Steelant, J., "Effect of a Compressibility Correction on Different Turbulence Models," Engineering Turbulence Modeling and Experiments 5, edited by W. Rodi and N. Fueyo, Elsevier, 2002, pp. 207-216.

${ }^{37}$ Oliver, A. B., Lillard, R. P., Blaisdell, G. A., and Lyrintzis, A. S., "Effects of Three-Dimensionality in Turbulent Compression Ramp Shock-Boundary Layer Interaction Computations," 46th AIAA Aerospace Sciences Meeting and Exhibit, Reno, NV, January 2008, AIAA 2008-720. 
${ }^{38}$ Oliver, A. B., Lillard, R. P., Schwing, A. M., Blaisdell, G. A., and Lyrintzis, A. S., "Assessment of Turbulent ShockBoundary Layer Interaction Computations Using the OVERFLOW code," 45th AIAA Aerospace Sciences Meeting and Exhibit, Reno, NV, January 2007, AIAA 2007-104.

${ }^{39}$ Oliver, A. B., Lillard, R. P., Blaisdell, G. A., and Lyrintzis, A. S., "Validation of High-Speec Turbulent Boundary layer and Shock-Boundary Layer Interaction Computations with the OVERFLOW Code," 44th AIAA Aerospace Sciences Meeting and Exhibit, Reno, NV, January 2006, AIAA 2006-894.

${ }^{40}$ Bell, J., "Transonic/Supersonic Wind Tunnel Testing of the NASA Crew Exploration Vehicle (CEV)," 45th AIAA Aerospace Sciences Meeting and Exhibit, 8 - 11 January 2007, Reno, NV, January 2007, AIAA Paper 2007-1006.

${ }^{41}$ Bell, J., "Test 5-CA Final Report," EG-CEV-06-19.

${ }^{42}$ Amaya, M. A. and Boone, A., "Calibration of the 11-By 11-Foot Transonic Wind Tunnel at the NASA Ames Research Center," 41st AIAA/ASME/SAE/ASEE Joint Propulsion Conference and Exhibit, Tucson, Arizona, July 10-13, 2005, July 2005, AIAA 2005-4277.

${ }^{43}$ Chan, W. M., Gomez, R. J., Rogers, S. E., and Buning, P. G., "Best Practices in Overset Grid Generation," 32nd AIAA Fluid Dynamics Conference, St. Louis, MO, June 2002, AIAA 2002-3191.

${ }^{44}$ Meakin, R. L., "Automatic Off-Body Grid Generation for Domains of Arbitrary Size," June 2001, AIAA $2001-2536$.

${ }^{45}$ Meakin, R. L., "Object X-rays for Cutting Holes in Composite Overset Structured Meshes," June 2001, AIAA $2001-2537$.

${ }^{46}$ Murray, N. and Hillier, R., "Hypersonic ShockWave/Turbulent Boundary layer Interactions in A Three-Dimensional Flow," 44th AIAA Aerospace Sciences Meeting and Exhibit, Reno, NV, January 2006, AIAA 2006-121.

${ }^{47}$ Murray, N. and Hillier, R., "Separated ShockWave/Turbulent Boundary layer Interactions at Hypersonic Speeds," 36th AIAA Fluid Dynamics Conference and Exhibit, San Francisco, CA, June 2006, AIAA 2006-3038.

${ }^{48}$ Murray, N., Three-Dimensional Turbulent Shock-Wave/Boundary Layer Interactions at Hypersonic Speeds, PhD Dissertation, Imperial College, University of London, 2007.

${ }^{49}$ Kussoy, M. and Horstman, K., "Documentation of two- and three-dimensional shock wave turbulent boundary layer interactions at Mach 8.2," Tech. Rep. NASA TM 103838, NASA Ames Research Center, May 1991.

${ }^{50}$ Speziale, C. G. and Xu, X. H., "Towards the development of second-order closure models for nonequilibrium turbulent flows," International Journal of Heat and Fluid FLow, , No. 17, 1996, pp. 238-244.

${ }^{51}$ Radhia, F., Marzougui, H., Jihene, Z., and Lili, T., "On a relaxation model for non-equilibrium flows', Comptes Rendus Mecanique, , No. 338, July 2010, pp. 355-360.

${ }^{52}$ Coakley, T. J., "Development of turbulence models for aerodynamic applications," AIAA 28th Fluid Dynamics Conference, June 29-July 2, 1997, Snowmass Village, CO, July 1997, AIAA 97-2009. 